\section{Women economic empowerment via cash transfer and microcredit programs is enough to decrease intimate partner violence? Evidence from a systematic review}

\author{
O empoderamento econômico das mulheres \\ através de programas de transferência de renda \\ e de microcrédito é suficiente para diminuir a \\ violência entre parceiros íntimos? Evidências \\ de uma revisão sistemática
}

\author{
¿El empoderamiento económico de las mujeres a \\ través de programas de transferencia de renta y \\ de microcréditos basta para disminuir la \\ violencia doméstica? Evidencias de \\ una revisión sistemática
}

Tatiana Henriques Leite 1 Claudia Leite de Moraes 1 Emanuele Souza Marques 1 Rosangela Caetano 1 José Ueleres Braga 1 Michael Eduardo Reichenheim ${ }^{1}$

doi: 10.1590/0102-311X00174818

\author{
Correspondence \\ T. H. Leite \\ Universidade do Estado do Rio de Janeiro. \\ Rua São Francisco Xavier 524, 7o andar, blocos D e E, \\ Rio de Janeiro, RJ 227750-033, Brasil. \\ henriques.tatiana@gmail.com \\ 1 Universidade do Estado do Rio de Janeiro, Rio de Janeiro, \\ Brasil.
}

Intimate Partner Violence; Government Programs; Empowerment; Women \begin{abstract}
opposite effect. The objective of this study was to investigate the influence of women's economic empowerment in MP and CTP on the risk of physical, psychological and sexual violence through a systematic review. Papers/documents selection was conducted by two researchers according to the following criteria: published in English, Portuguese or Spanish; primary data; assessing the effect of MP or CTP on IPV; in heterosexual couples; on women beneficiaries of the intervention; using a comparator group eligible for an MP or CTP; and focusing on risk IPV as the outcomes. Our results showed that the impact of $M P$ are mixed when it comes to physical and physical/sexual violence. Even so, the review suggests that the effect of MP on sexual violence is trivial or nonexistent. Regarding the impact of CTPs, the present study showed that the effects on physical, physical/sexual, psychological, and sexual violence were also heterogeneous. Women more empowered and with some autonomy could be at risk. Despite that, participation in the empowerment program should be encouraged for poor women and families. However, parallel interventions to lead with IPV should be addressed to the main actions to reduce the risk of increasing IPV prevalence in certain scenarios.

Intimate partner violence (IPV) is a worldwide public health problem. Many proposals aiming to eliminate its occurrence include the empowerment
of women through their socio-economic development. In this context, some studies suggested that microcredit programs (MP) and cash transfer programs
\end{abstract}

(n)




\section{Introduction}

Intimate partner violence (IPV) is an important public health problem worldwide due to its high prevalence and severe consequences not only for the individuals and families involved but also to society at large 1 . The estimated prevalence of IPV varies between $16.3 \%$ and $65.6 \%$, with the highest values found in South Asia (41.7\%), the Andean region of Latin America (40.6\%) and central SubSaharan Africa (65.6\%) 1. The consequences of IPV are massive, involving mental, physical and sexual problems, low professional productivity, and offer several barriers for victims to engage in normal, flourishing social activities 2 .

Women's economic empowerment, defined here as the ability to generate independent income 3 , is considered a key factor in the prevention of IPV 4,5,6. Programs based on microcredit or cash transfer aimed at eradicating poverty have thus been regarded as potential tools for reducing IPV. The main purpose of both types of program is to break the cycle of poverty by bringing women into the labor market - microcredit programs (MP) 7 - or by monthly transfers of fixed benefits to families in extremely vulnerable situations, also known as cash transfer programs (CTP) 8. CTP may be nonconditional when there are no restrictions to a payee's permanence in the program, or conditional, when child health and educational actions are required from the beneficiary for the inclusion and continuity in receiving benefits 8 .

Different theoretical frameworks have been used for trying to predict the consequences of women's economic empowerment on IPV. Tauchen et al. ${ }^{9}$, for instance, indicate that, depending on the context, IPV could either increase or decrease following an improvement in women's economic status. This model asserts that the increase of women's income raises their bargaining power within the marriage. In certain conservative gender-related contexts, this increases women's participation in the decision-making process within the domestic sphere, making marriage power relations more equitable, thus leading to a reduction in IPV. In other contexts, these increases in women's income could shift power relations towards the women, increasing IPV when men perceive that their authority within the family is fading following their relatively diminished participation as the main bread-winner 9 .

According to Farmer \& Tiefenthaler 10, raising women's income should necessarily lead to less IPV due to the decrease in their economic dependence on the partner. Reducing the financial gap lowers the tendency to tolerate frustrations and dissatisfaction in marriage, including IPV. In the opposite direction, Block \& Rao ${ }^{11}$ proposed that increasing women's income will further encourage a violent partner using violence as a strategy to extract financial resources from the victim.

Although these models help in building a rationale for the relationship between women's economic empowerment and IPV, there is no established consensus. Empirical evidence goes in the same direction. While some studies have shown MP protecting women against IPV 12,13,14, others do not detect this effect. There is also research showingthat women participating in an MP are more prone to be involved in IPV 15,16. The same controversy is detected in the literature assessing the effect of CTP 17,18 .

This lack of consensus has sparked a number of systematic reviews 19,20,21. The first was published in 2009 and aimed to identify whether women's economic and social empowerment was associated with a lower risk of violence against women. Vyas \& Watts 19 concluded that economic autonomy has a protective effect in almost all situations. However, they also noted that in specific contexts such as extreme poverty and low education, a woman's financial independence may in fact increase the risk of victimization. Although commendable for providing a first overview on the subject, this study involved only journals indexed in a single database (MEDLINE) and restricted to papers published in English. The second review was published in 201520 to assess the effect of economic and/or social interventions on IPV. Based on a qualitative summary of the literature, the authors found that overall, interventions lead to less IPV and controlling behaviors of partners. Although this review improved on the former, its scope remained narrow to the extent that it was still confined to two databases (MEDLINE and Web of Science) and English-only papers. Moreover, only one of the 16 identified studies examined the effects of CTP.

The most recent review 22, published in 2017, aimed to undertake a comprehensive review of published papers on the impact of economic interventions sought to prevent IPV and HIV risk behaviors. 
The review was once again restricted to articles written in English between January 2000 and January 2015 in three databases (MEDLINE, Web of Science and EbscoHost). Google Scholar was used to identify the related gray literature. In some studies, CTP failed to show any effect on IPV. In others, the initiative decreased violence. Microcredit interventions also entailed mixed outcomes, with some studies showing increase, decrease and no effect at all on IPV. Interventions combining microcredit and gender transformative interventions tended to have more positive outcomes, reducing IPV. However, the results presented were grouped only by the type of intervention, which precluded identifying the programs' impacts according to different types of violence 22 . The literature selection strategies were also a limitation, as they were not able to identify important papers uncovered in previous reviews.

Given these shortcomings, further studies involving more comprehensive summaries are required. To this end, this paper provides a new systematic review, offering a broader perspective, both in covering a broader range of databases, languages, and economic empowerment programs, and in distinguishing between occurrence setting and types of IPV. Specifically, we investigate the effects of CTP and MP on the occurrence of physical, psychological and sexual IPV against women.

\section{Methods}

\section{Protocol and search strategy}

The study protocol was registered in the PROSPERO (2016: CRD42016035980). Nine databases were accessed, namely, MEDLINE, LILACS, Cochrane, Embase, Science Direct, PsycINFO, Scopus, WHOLIS, and Open Gray. The last two relate specifically to the related "gray literature". The search was conducted on MEDLINE without language restriction and adapted for the other databases, according to the following strategy: ((“"Domestic Violence”[Mesh] OR "Battered Women”[Mesh] OR "Spouse Abuse"[Mesh] OR "intimate partner violence" OR "domestic violence" OR "spouse abuse" OR "battered woman" OR "wife abuse" OR "family violence" OR "partner violence”))) AND (((“Income”[Mesh] OR "small business"[Mesh] OR "cash transfer" OR "public transfer" OR "micro-credit" OR "micro credit" OR "microcredit" OR "income generation" OR "income generating" OR "economic empowerment" OR "cooperatives" OR "micro-finance" OR "micro finance" OR "micro-enterprise" OR "micro enterprise" OR "microenterprise" OR "small business" OR "business, small" OR "small loans" OR "micro loans" OR "microloans" OR "micro-loans" OR "incomes" OR "income generation programs" OR "income generation program" OR "program, income generation" OR "programs, income generation" OR "savings" OR "income distribution" OR "distribution, income" OR "distributions, income" OR "income distributions" OR "family income"))). The search strategies used in the different databases may be provided by authors upon request. Manual searches were also conducted in the reference sections of all selected studies. Database search took place in January 2018.

\section{Selection process}

The selection process was independent (T.H.L. and E.S.M.). Papers/documents were first selected on titles and abstracts, according to the following inclusion criteria: publications in English, Portuguese or Spanish; papers/documents related to MP or CTP and IPV; and studies on heterosexual couples. The next step involved reading the text in full, and applying the following eligibility criteria: papers/ documents with primary data; women as the beneficiary of the intervention; presence of a comparator group eligible for an MP or CTP; and estimates of prevalence, incidence and relative risk/odds ratio related to physical, psychological or sexual IPV. All inconsistencies regarding the inclusion of papers/documents were discussed by the two researchers until a consensus was reached. 


\section{Data extraction}

Extracted data comprised the paper's identification, demographic characteristics of the study participants and the following methodological features: sample size, intervention strategy, measurement tools used to tap IPV, data collection period, effect measures and analysis strategies employed. The extraction was also carried out independently (T.H.L and E.S.M). Disagreements were solved by referencing back to the original publication and consensus.

\section{Data analysis}

The literature synthesis was implemented according to the type of intervention, study design and type of violence. When the subgroups formed by these variables entailed more than five original papers, the stratification also encompassed physical and physical/sexual baseline IPV prevalence; place of living (rural vs. urban); and religion.

Assessment of study quality followed the recommendations found in The Joanna Briggs Institute Reviewers Manual 2014(MAStARI Critical Appraisal Checklist), for surveys/case series and experimental studies 23. The MAStARI is the most adequate tool for observational studies, which comprise the majority reviewed. Checklists for experimental and survey/case studies encompass ten and nine items, respectively (Tables 1 and 2). In observational checklist, since the sixth item is only appropriate for case series and none of the selected studies were of this type, it was excluded from the checklist. In the same questionnaire, item seven was also adapted to seize the response rate of the population eligible to participate in the study. A response rate equal to or above $85 \%$ was considered appropriate 24 .

Item Q1 (Truly Randomization) and Q4 (Intention to Treat Analysis) were chosen for the methodologic quality evaluation of experimental studies; and Q1 (Random or Pseudo-Random Sample) and Q9 (Statistical Analysis) when dealing with observational ones. The latter criterion considered the women's age and educational status as the minimum set of confounders for judging the appropriateness of a given analysis.

Initially, data analysis and interpretation considered all selected original papers, but we also performed a subgroup evaluation considering only the investigations without methodological limitations on the criteria mentioned above.

Table 1

Critical appraisal results for randomized controlled trials according to the MAStARI Critical Appraisal Checklist.

\begin{tabular}{|c|c|c|c|c|c|c|c|c|c|c|}
\hline Reference & Q1 & Q2 & Q3 & Q4 & Q5 & Q6 & Q7 & Q8 & Q9 & Q10 \\
\hline \multicolumn{11}{|l|}{ Microcredit } \\
\hline Green et al. 28 (2015) & Y & $\mathrm{N}$ & $\mathrm{N}$ & Y & $\mathrm{N}$ & $\mathrm{N}$ & $\mathrm{Y}$ & Y & $\mathrm{N}$ & Y \\
\hline Pronyk et al. 25 (2006) & $\mathrm{Y}$ & $\mathrm{U}$ & $\mathrm{U}$ & $\mathrm{N}$ & $U$ & $\mathrm{Y}$ & $\mathrm{Y}$ & $\mathrm{Y}$ & $\mathrm{Y}$ & Y \\
\hline Tsai et al. 29 (2016) & $U$ & $\mathrm{~N}$ & $\mathrm{~N}$ & $\mathrm{Y}$ & $U$ & $\mathrm{~N}$ & Y & $\mathrm{Y}$ & $\mathrm{Y}$ & Y \\
\hline \multicolumn{11}{|l|}{ Cash transfer } \\
\hline Angelucci 6 (2008) & $\mathrm{Y}$ & $U$ & $U$ & $U$ & $U$ & Y & Y & Y & $U$ & $\mathrm{Y}$ \\
\hline Hidrobo \& Fernald 18 (2013) & $\mathrm{Y}$ & $U$ & $U$ & $U$ & $U$ & $Y$ & $Y$ & $Y$ & $Y$ & $Y$ \\
\hline Pettifor et al. 44 (2016) & Y & $\mathrm{N}$ & $\mathrm{Y}$ & $\mathrm{Y}$ & $\mathrm{Y}$ & Y & Y & $\mathrm{Y}$ & $U$ & Y \\
\hline
\end{tabular}

N: no; U: unclear; Y: yes.

Note: Items: Q1 - Truly Randomization; Q2 - Participants Blinded; Q3 - Allocation Concealment to Groups; Q4 - Intention to Treat Analysis; Q5 - Outcome Evaluator Blinded to Allocation; Q6 - Control and Treatment are Comparable; Q7 - Groups treated Identically Other than Intervention; Q8 - Outcomes Measured in the Same Way for All Groups; Q9 - Outcome Measurement; Q10 - Statistical Analysis. 


\section{Table 2}

Critical appraisal results for included studies using the MAStARI Critical Appraisal Checklist - descriptive/case series studies.

\begin{tabular}{|c|c|c|c|c|c|c|c|c|}
\hline Reference & Q1 & Q2 & Q3 & Q4 & Q5 & Q7 & Q8 & Q9 \\
\hline \multicolumn{9}{|l|}{ Microcredit } \\
\hline Ahmed 41 (2005) & $\mathrm{N}$ & $\mathrm{Y}$ & Y & $\mathrm{N}$ & $\mathrm{N}$ & $U$ & $\mathrm{Y}$ & Y \\
\hline Bajracharya \& Amin 35 (2013) & Y & $\mathrm{Y}$ & Y & Y & $\mathrm{Y}$ & Y & $U$ & Y \\
\hline Bates et al. 31 (2004) & $\mathrm{N}$ & $\mathrm{Y}$ & Y & Y & $\mathrm{N}$ & Y & $u$ & Y \\
\hline Bhuiya et al. 15 (2003) & $\mathrm{N}$ & Y & Y & $U$ & $\mathrm{~N}$ & $u$ & $\mathrm{Y}$ & N \\
\hline Cepeda et al. 40 (2017) & $u$ & Y & Y & $\mathrm{N}$ & Y & $U$ & $\mathrm{Y}$ & Y \\
\hline Chin 34 (2012) & $\mathrm{N}$ & $\mathrm{Y}$ & Y & $\mathrm{N}$ & Y & $\mathrm{N}$ & $U$ & Y \\
\hline Dalal et al. 16 (2013) & $\mathrm{N}$ & $\mathrm{Y}$ & Y & $\mathrm{Y}$ & Y & N & $\mathrm{Y}$ & $u$ \\
\hline Dutt et al. 14 (2015) & $\mathrm{Y}$ & $\mathrm{N}$ & Y & $\mathrm{Y}$ & Y & Y & $\mathrm{Y}$ & N \\
\hline Hadi 32 (2000) & $U$ & $\mathrm{Y}$ & Y & N & Y & Y & $\mathrm{Y}$ & $u$ \\
\hline Hadi 13 (2005) & $u$ & $\mathrm{Y}$ & $\mathrm{Y}$ & $\mathrm{N}$ & Y & Y & $\mathrm{Y}$ & $u$ \\
\hline Hasan et al. 42 (2014) & $\mathrm{N}$ & Y & Y & $U$ & $\mathrm{~N}$ & $u$ & $\mathrm{Y}$ & Y \\
\hline Karim \& Law 43 (2016) & $\mathrm{Y}$ & $\mathrm{N}$ & Y & Y & $\mathrm{N}$ & N & $U$ & Y \\
\hline Kim et al. 37 (2009) & Y & $\mathrm{Y}$ & Y & Y & Y & $U$ & $\mathrm{Y}$ & Y \\
\hline Koening et al. 33 (2003) & $u$ & $\mathrm{Y}$ & Y & N & N & Y & $u$ & Y \\
\hline Murshid et al. 38 (2015) & $\mathrm{Y}$ & $\mathrm{Y}$ & Y & Y & $\mathrm{N}$ & $\mathrm{N}$ & $U$ & Y \\
\hline Murshid 36 (2016) & Y & $\mathrm{Y}$ & Y & Y & $\mathrm{N}$ & $\mathrm{N}$ & $u$ & Y \\
\hline Naved \& Persson 26 (2005) & $\mathrm{Y}$ & $\mathrm{Y}$ & $\mathrm{Y}$ & Y & $\mathrm{N}$ & $U$ & $\mathrm{Y}$ & Y \\
\hline Sarker et al. 30 (2016) & $\mathrm{Y}$ & $\mathrm{N}$ & Y & U & Y & $U$ & $U$ & Y \\
\hline Schuler et al. 12 (1996) & $\mathrm{N}$ & $u$ & Y & N & N & Y & $u$ & Y \\
\hline Vyas et al. 39 (2015) & Y & $\mathrm{Y}$ & Y & Y & N & Y & $u$ & N \\
\hline \multicolumn{9}{|l|}{ Cash transfer } \\
\hline Aísa 49 (2014) & Y & $U$ & Y & $\mathrm{N}$ & Y & $U$ & $U$ & N \\
\hline Bobonis et al. 17 (2013) & Y & Y & Y & $u$ & Y & $\mathrm{N}$ & $u$ & Y \\
\hline Bobonis et al. 48 (2015) & $\mathrm{Y}$ & $\mathrm{Y}$ & Y & U & N & $\mathrm{N}$ & $U$ & Y \\
\hline Perova 47 (2010) & $\mathrm{Y}$ & Y & Y & N & $\mathrm{N}$ & $\mathrm{N}$ & $U$ & Y \\
\hline Rivera et al. 46 (2006) & $\mathrm{N}$ & $\mathrm{Y}$ & Y & N & Y & Y & $u$ & Y \\
\hline Tolman \& Rosen 45 (2001) & Y & Y & Y & $\mathrm{Y}$ & $\mathrm{N}$ & Y & $u$ & N \\
\hline
\end{tabular}

$\mathrm{N}$ : no; U: unclear; Y: yes.

Note: Items: Q1 - Random or Pseudo-Random Sample; Q2 - Criteria for Inclusion Defined; Q3 - Strategies to Deal with Confounding; Q4 - Outcomes Assess; Q5 - Comparisons Between the Groups; Q6 - Not Applicable; Q7 - Response Rate; Q8 - Outcome Measurement; Q9 - Statistical Analysis.

\section{Results}

\section{Study selection}

As shown in Figure 1, 22,685 non-duplicate references were recovered. Applying the inclusion and the eligibility criteria, 32 studies were selected for the full-text review. While reading the full papers, two were identified as having the same content 5,25, and another two shared the same dataset although involving different confounding variables in the statistical analysis 26,27. To avoid bias from duplication, only the first publication in both situations was included in the ensuing analyses 25,26. 

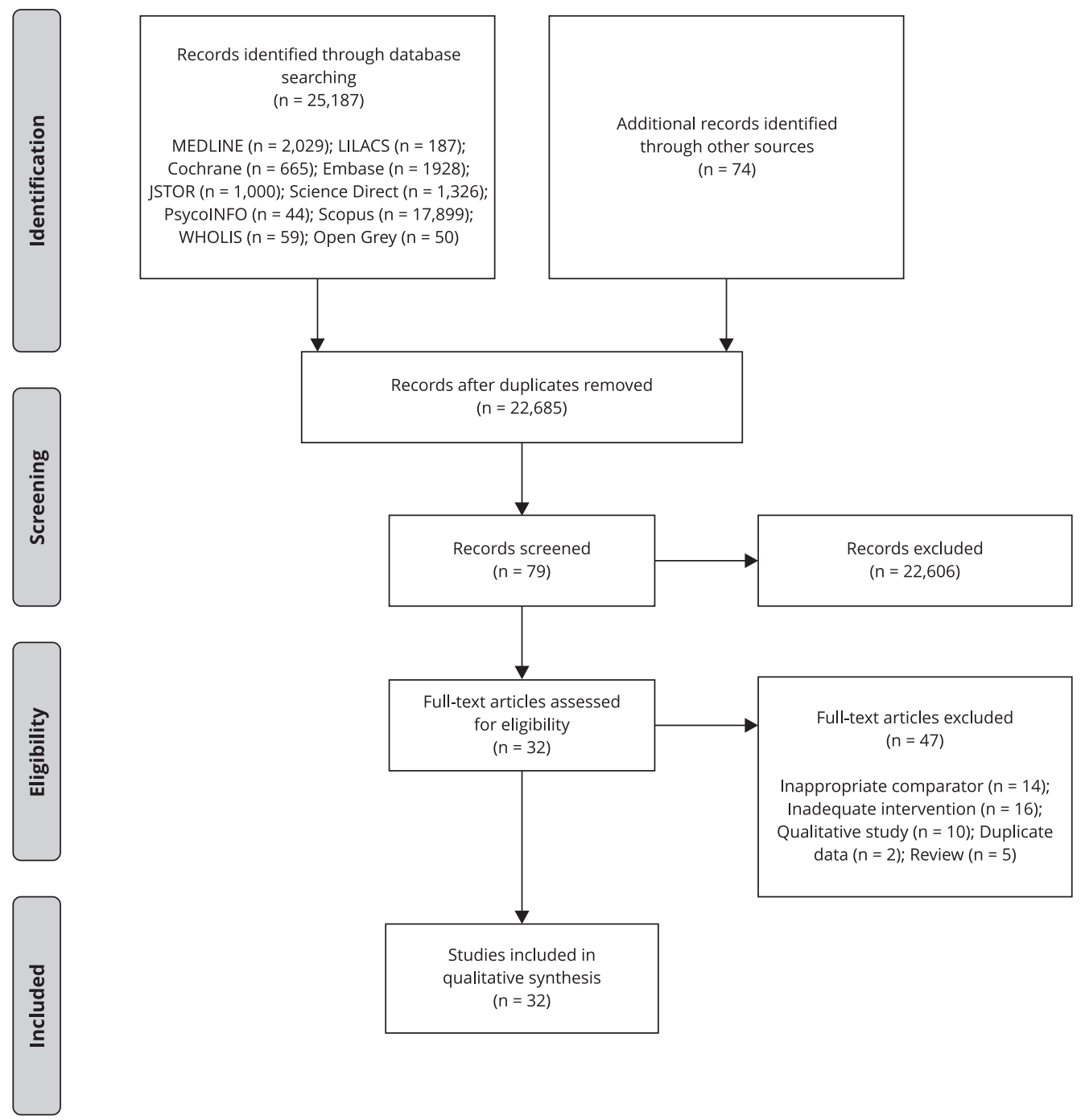

Source: Moher et al. 56. For more information, visit http://www.prisma-statement.org.

\section{Study characteristics}

Table 3 shows the general and methodological characteristics of the studies. All were published between 1996 and 2017, particularly after 2010. Research was conducted in Bangladesh (16), Mongolia (1), Tanzania (2), South Africa (3), Uganda (1), United States (1), Guatemala (1), Ecuador (1), Mexico (5) and Peru (1). Twenty-six were surveys and six were randomized controlled trials. Twenty-three studies covered the effects of MP while nine focused on CTP. Most studies concerned married or ever-married women. Only five used specific population: men (2), women with disability (1), school girls (1) and sex workers (1). The comparator groups were composed of women who did not participate in the economic empowerment programs albeit potentially eligible. Physical violence was the most studied type of violence, followed by a combination of physical and sexual violence. The 
Table 3

Identification, participants and main methodological characteristics of the studies selected for the systematic review.

\begin{tabular}{|c|c|c|c|c|c|c|c|}
\hline References & $\begin{array}{l}\text { Country } \\
\text { (Year) }\end{array}$ & $\begin{array}{l}\text { Study design/ } \\
\text { Participants/ } \\
\text { Sample size }\end{array}$ & $\begin{array}{c}\text { Baseline } \\
\text { prevalence of } \\
\text { IPV }\end{array}$ & Intervention & $\begin{array}{l}\text { Control } \\
\text { intervention }\end{array}$ & $\begin{array}{l}\text { Type of IPV/ } \\
\text { Measurement } \\
\text { tool/Recall } \\
\text { period }\end{array}$ & $\begin{array}{l}\text { Confounding and } \\
\text { effect modification } \\
\text { variables }\end{array}$ \\
\hline \multicolumn{8}{|l|}{ Microcredit } \\
\hline \multicolumn{8}{|l|}{ RCT } \\
\hline $\begin{array}{l}\text { Green et } \\
\text { al. } 28\end{array}$ & $\begin{array}{l}\text { Uganda } \\
\text { (2015) }\end{array}$ & $\begin{array}{c}\text { Cluster RCT; } \\
\text { rural villages, } \\
\text { marriage or } \\
\text { single women } \\
\text { (14-30 years); } \\
\text { Christians: } 85 \% ; \\
\text { sample size: } \\
\text { 1,546 }\end{array}$ & Not informed & $\begin{array}{l}\text { Microcredit + } \\
\text { business skills } \\
\text { training + follow- } \\
\text { up support }\end{array}$ & $\begin{array}{c}\text { Microcredit } \\
\text { non-members }\end{array}$ & $\begin{array}{c}\text { Physical, } \\
\text { psychological } \\
\text { violence and } \\
\text { control behavior; } \\
14 \text { isolated } \\
\text { questions about } \\
\text { IPV; recall period: } \\
\text { last } 8 \text { months }\end{array}$ & $\begin{array}{l}\text { Employment, started } \\
\text { enterprise since } \\
\text { baseline, currently } \\
\text { doing business, } \\
\text { average work hour } \\
\text { per week, income, } \\
\text { monthly cash earnings, } \\
\text { durable assets, non- } \\
\text { durable consumption, } \\
\text { perceptions of } \\
\text { women's autonomy, } \\
\text { self-reported } \\
\text { autonomy, partner } \\
\text { relationship index }\end{array}$ \\
\hline $\begin{array}{l}\text { Pronyk et } \\
\text { al. } 25\end{array}$ & $\begin{array}{l}\text { South Africa } \\
\text { (2006) }\end{array}$ & $\begin{array}{c}\text { Cluster RCT; } \\
\text { rural villages, } \\
\text { marriage } \\
\text { women (> 50\%); } \\
\text { sample size: } 860\end{array}$ & $\begin{array}{c}\text { Physical or/ } \\
\text { and sexual IPV } \\
\text { prevalence: } \\
11 \% \\
\text { (intervention } \\
\text { group) and 9\% } \\
\text { (comparison } \\
\text { group) in the } \\
\text { last year }\end{array}$ & $\begin{array}{l}\text { IMAGE *: credit } \\
\quad+\text { training } \\
\text { + lecturer } \\
\text { education + } \\
\text { ten sessions } \\
\text { about gender } \\
\text { education }\end{array}$ & $\begin{array}{c}\text { Microcredit } \\
\text { non-members }\end{array}$ & $\begin{array}{l}\text { Physical and } \\
\text { sexual IPV } \\
\text { combined. WHO's } \\
\text { questionnaire **; } \\
\text { recall period: last } \\
12 \text { months }\end{array}$ & $\begin{array}{l}\text { Women's age, village } \\
\text { pair, marital status, } \\
\text { lifetime experience } \\
\text { of intimate-partner } \\
\text { violence by current } \\
\text { partner at baseline }\end{array}$ \\
\hline Tsai et al. 29 & $\begin{array}{l}\text { Mongolia } \\
\text { (2016) }\end{array}$ & $\begin{array}{l}\text { Individual RCT; } \\
\text { urban villages, } \\
\text { women engage } \\
\text { in sex work; } \\
\text { Buddhists: } 53 \% ; \\
\text { sample size: } 107\end{array}$ & $\begin{array}{l}\text { Physical or/ } \\
\text { and sexual } \\
\text { prevalence: } \\
48 \% \text { in control } \\
\text { group, 40,4\% } \\
\text { in microcredit } \\
\text { group }\end{array}$ & $\begin{array}{l}\text { Credit + } 4 \\
\text { sessions } \\
\text { intervention } \\
\text { about sex } \\
\text { protection, } \\
\text { safety and } \\
\text { sexual risk } \\
\text { reduction }\end{array}$ & $\begin{array}{c}\text { Microcredit } \\
\text { non-members }\end{array}$ & $\begin{array}{l}\text { Physical, sexual } \\
\text { and physical } \\
\text { and sexual } \\
\text { combined IPV; CTS } \\
\text { questionnaire ***; } \\
\text { recall period: last } \\
3 \text { and } 6 \text { months }\end{array}$ & No adjustment \\
\hline
\end{tabular}

(continues) 
Table 3 (continued)

\begin{tabular}{|c|c|c|c|c|c|c|c|}
\hline References & $\begin{array}{l}\text { Country } \\
\text { (Year) }\end{array}$ & $\begin{array}{l}\text { Study design/ } \\
\text { Participants/ } \\
\text { Sample size }\end{array}$ & $\begin{array}{c}\text { Baseline } \\
\text { prevalence of } \\
\text { IPV }\end{array}$ & Intervention & $\begin{array}{c}\text { Control } \\
\text { intervention }\end{array}$ & $\begin{array}{c}\text { Type of IPV/ } \\
\text { Measurement } \\
\text { tool/Recall } \\
\text { period }\end{array}$ & $\begin{array}{l}\text { Confounding and } \\
\text { effect modification } \\
\text { variables }\end{array}$ \\
\hline \multicolumn{8}{|l|}{ Microcredit } \\
\hline \multicolumn{8}{|l|}{ Surveys } \\
\hline Ahmed 41 & $\begin{array}{l}\text { Bangladesh } \\
\text { (2005) }\end{array}$ & $\begin{array}{c}\text { Rural village, } \\
\text { ever-marriage } \\
\text { women (15-49 } \\
\text { years); Islamics: } \\
\text { 86,6\%; sample } \\
\text { size: } 2,044\end{array}$ & $\begin{array}{l}\text { Prevalence } \\
\text { of physical or } \\
\text { psychological } \\
\text { IPV: } 14,5 \%\end{array}$ & $\begin{array}{c}\text { BRAC \#: } \\
\text { non-formal } \\
\text { education, skill } \\
\text { development } \\
\text { training, and } \\
\text { collateral-free } \\
\text { loan for income } \\
\text { generating } \\
\text { activities }\end{array}$ & $\begin{array}{l}\text { Eligible BRAC } \\
\text { non-members }\end{array}$ & $\begin{array}{l}\text { Physical and } \\
\text { psychological } \\
\text { violence; isolated } \\
\text { questions about } \\
\text { IPV; recall period: } \\
\text { last } 4 \text { months }\end{array}$ & $\begin{array}{l}\text { Women's age, } \\
\text { education, contribution } \\
\text { to household income, } \\
\text { currently-alive children, } \\
\text { age and schooling } \\
\text { of household head, } \\
\text { poverty status }\end{array}$ \\
\hline $\begin{array}{l}\text { Bajracharya } \\
\text { \& Amin } 35\end{array}$ & $\begin{array}{l}\text { Bangladesh } \\
\text { (2013) }\end{array}$ & $\begin{array}{c}\text { Rural and } \\
\text { urban villages, } \\
\text { ever-marriage } \\
\text { women (15-49 } \\
\text { years); Muslims: } \\
\text { 90\%; sample } \\
\text { size: 4,195 }\end{array}$ & $\begin{array}{c}\text { Prevalence } \\
\text { of physical or } \\
\text { sexual violence } \\
\text { in microcredit } \\
\text { group: } 28 \% \text {; } \\
\text { microcredit } \\
\text { non-members: } \\
21,5 \%\end{array}$ & $\begin{array}{c}\text { Grameen } \\
\text { Bank \#, BRAC\#, } \\
\text { ASA \#, Proshika } \\
\text { \# and others } \\
\text { microcredit } \\
\text { programs }\end{array}$ & $\begin{array}{c}\text { Microcredit } \\
\text { non-members }\end{array}$ & $\begin{array}{l}\text { Physical and } \\
\text { sexual violence; } \\
\text { CTS adaptation } \\
\text { (8 questions) }{ }^{\star \star *} \text {; } \\
\text { recall period: last } \\
12 \text { months }\end{array}$ & $\begin{array}{l}\text { Women's age and } \\
\text { education, age at first } \\
\text { marriage, spouse's } \\
\text { education, household } \\
\text { size, household sex, } \\
\text { socioeconomic status, } \\
\text { district, rural/urban }\end{array}$ \\
\hline Bates et al. 31 & $\begin{array}{l}\text { Bangladesh } \\
\text { (2004) }\end{array}$ & $\begin{array}{c}\text { Rural villages, } \\
\text { marriage } \\
\text { women (<50 } \\
\text { years); Muslims: } \\
\text { 96\%; sample } \\
\text { size: 1,211 }\end{array}$ & $\begin{array}{l}\text { Prevalence of } \\
\text { physical IPV: } \\
35 \%\end{array}$ & Microcredit & $\begin{array}{c}\text { Microcredit } \\
\text { non-members }\end{array}$ & $\begin{array}{l}\text { Physical IPV; } \\
\text { adaptation } \\
\text { of the WHO's } \\
\text { questionnaire } \\
\text { ** (6 questions); } \\
\text { recall period: last } \\
12 \text { months }\end{array}$ & $\begin{array}{l}\text { Women's age, } \\
\text { education, and } \\
\text { contribution to } \\
\text { household income, } \\
\text { registered marriage, } \\
\text { dowry agreement, } \\
\text { household } \\
\text { socioeconomic status }\end{array}$ \\
\hline $\begin{array}{l}\text { Bhuiya et } \\
\text { al. } 15\end{array}$ & $\begin{array}{l}\text { Bangladesh } \\
\text { (2003) }\end{array}$ & $\begin{array}{c}\text { Rural village, } \\
\text { ever-marriage } \\
\text { women (17-70 } \\
\text { years); Muslims: } \\
\text { 91\%; sample } \\
\text { size: } 189\end{array}$ & $\begin{array}{l}\text { Lifetime } \\
\text { prevalence } \\
\text { of physical } \\
\text { IPV: 55,8\%; } \\
\text { psychological } \\
\text { IPV: } 66,8 \%\end{array}$ & $\begin{array}{l}\text { Samities \# } \\
\text { microcredit }\end{array}$ & $\begin{array}{c}\text { Microcredit } \\
\text { non-members }\end{array}$ & $\begin{array}{c}\text { Physical and } \\
\text { psychological } \\
\text { violence; isolated } \\
\text { question about } \\
\text { IPV; recall period: } \\
\text { in life }\end{array}$ & Husband's age \\
\hline $\begin{array}{l}\text { Cepeda et } \\
\text { al. } 40\end{array}$ & $\begin{array}{c}\text { Guatemala } \\
\text { (2017) }\end{array}$ & $\begin{array}{c}\text { Rural village, } \\
\text { marriage } \\
\text { women (80\%); } \\
\text { Catholics: } 95 \% \text {; } \\
\text { sample size: } 883\end{array}$ & $\begin{array}{l}\text { Prevalence of } \\
\text { any type of } \\
\text { IPV: } 12,7 \% \text { in } \\
\text { microcredit } \\
\text { group; } 28,5 \% \text { in } \\
\text { non-microcredit } \\
\text { group }\end{array}$ & Microcredit & $\begin{array}{c}\text { Microcredit } \\
\text { non-members }\end{array}$ & $\begin{array}{l}\text { Control behavior, } \\
\text { economic and } \\
\text { psychological } \\
\text { violence; } 7 \\
\text { isolated questions } \\
\text { about IPV; recall } \\
\text { period not } \\
\text { available }\end{array}$ & $\begin{array}{l}\text { Women's age, number } \\
\text { of children, education, } \\
\text { wage, women main } \\
\text { breadwinner }\end{array}$ \\
\hline
\end{tabular}

(continues) 
Table 3 (continued)

\begin{tabular}{|c|c|c|c|c|c|c|c|}
\hline References & $\begin{array}{l}\text { Country } \\
\text { (Year) }\end{array}$ & $\begin{array}{l}\text { Study design/ } \\
\text { Participants/ } \\
\text { Sample size }\end{array}$ & $\begin{array}{c}\text { Baseline } \\
\text { prevalence of } \\
\text { IPV }\end{array}$ & Intervention & $\begin{array}{c}\text { Control } \\
\text { intervention }\end{array}$ & $\begin{array}{c}\text { Type of IPV/ } \\
\text { Measurement } \\
\text { tool/Recall } \\
\text { period }\end{array}$ & $\begin{array}{l}\text { Confounding and } \\
\text { effect modification } \\
\text { variables }\end{array}$ \\
\hline \multicolumn{8}{|l|}{ Microcredit } \\
\hline \multicolumn{8}{|l|}{ Surveys } \\
\hline Chin 34 & $\begin{array}{c}\text { Bangladesh } \\
\text { (2012) }\end{array}$ & $\begin{array}{c}\text { Rural village, } \\
\text { ever-marriage } \\
\text { women; } \\
\text { Muslims: 80\%; } \\
\text { sample size: } \\
1,843\end{array}$ & $\begin{array}{c}\text { Prevalence } \\
\text { of physical or } \\
\text { sexual IPV: } 38 \% \\
\text { in microcredit } \\
\text { group, 37\% in } \\
\text { non-microcredit } \\
\text { group }\end{array}$ & $\begin{array}{c}\text { Grameen } \\
\text { Bank \#, BRAC \#, } \\
\text { BRDB \# and } \\
\text { Asha \# }\end{array}$ & $\begin{array}{c}\text { Microcredit } \\
\text { non-members }\end{array}$ & $\begin{array}{c}\text { Physical and } \\
\text { sexual violence; } 6 \\
\text { isolated questions } \\
\text { about IPV; recall } \\
\text { period: last } 12 \\
\text { months }\end{array}$ & $\begin{array}{c}\text { Women's and } \\
\text { husband's age and } \\
\text { education, number of } \\
\text { sons at home, number } \\
\text { of children, women's } \\
\text { number of unions, } \\
\text { religion, land size, } \\
\text { distance from village } \\
\text { to a health center, } \\
\text { distance from village to } \\
\text { district headquarters, } \\
\text { telephone and } \\
\text { television service, } \\
\text { family planning, shop } \\
\text { presence in village, } \\
\text { indicator of satellite, } \\
\text { health in village }\end{array}$ \\
\hline Dalal et al. 16 & $\begin{array}{c}\text { Bangladesh } \\
\text { (2013) }\end{array}$ & $\begin{array}{c}\text { Urban and } \\
\text { rural villages, } \\
\text { ever-marriage } \\
\text { women (15-49 } \\
\text { years); Muslims: } \\
\text { 90\%; sample } \\
\text { size: 4,464 }\end{array}$ & $\begin{array}{l}\text { Prevalence of } \\
\text { physical or/and } \\
\text { sexual IPV: } 51 \% \\
\text { in the past year }\end{array}$ & $\begin{array}{l}\text { Grameen } \\
\text { Bank \#, BRDB \#, } \\
\text { BRAC \#, Asha \#, } \\
\text { Proshika \# and } \\
\text { any microcredit } \\
\text { organization }\end{array}$ & $\begin{array}{c}\text { Microcredit } \\
\text { non-members }\end{array}$ & $\begin{array}{l}\text { Physical, sexual } \\
\text { and physical } \\
\text { and/or sexual } \\
\text { combined; CTS } \\
\text { questionnaire ***; } \\
\text { recall period: last } \\
12 \text { months }\end{array}$ & $\begin{array}{c}\text { Age, residence, } \\
\text { education, religion, } \\
\text { wealth index. Effect } \\
\text { modification: spousal } \\
\text { equity and women's } \\
\text { education }\end{array}$ \\
\hline Dutt et al. 14 & $\begin{array}{c}\text { Tanzania } \\
\text { (2016) }\end{array}$ & $\begin{array}{l}\text { Rural village, } \\
\text { marriage } \\
\text { women, physical } \\
\text { (30-52 years); } \\
\text { Christians: } \\
\text { 61,4\%; sample } \\
\text { size: } 224\end{array}$ & $\begin{array}{l}\text { Prevalence of } \\
\text { physical IPV: } \\
\text { 32\% in the past } \\
\text { year }\end{array}$ & $\begin{array}{c}\text { MWEDO \#: } \\
\text { education, } \\
\text { health services, } \\
\text { enterprise } \\
\text { development, } \\
\text { and through } \\
\text { promotion of } \\
\text { human and } \\
\text { cultural rights }\end{array}$ & $\begin{array}{c}\text { Microcredit } \\
\text { non-members }\end{array}$ & 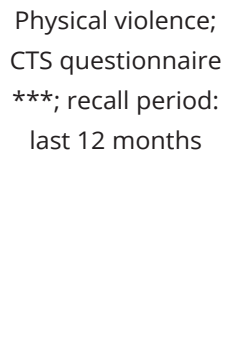 & $\begin{array}{c}\text { Financial decision } \\
\text { making, partner } \\
\text { control, depression, } \\
\text { self-esteem }\end{array}$ \\
\hline Hadi 32 & $\begin{array}{c}\text { Bangladesh } \\
\text { (2000) }\end{array}$ & $\begin{array}{l}\text { Rural villages, } \\
\text { marriage } \\
\text { women }(<50 \\
\text { years); sample } \\
\text { size: } 500\end{array}$ & $\begin{array}{l}\text { Prevalence } \\
\text { sexual IPV: } \\
26,8 \% \text { in the } \\
\text { past year }\end{array}$ & $\begin{array}{l}\text { Microcredit } \\
\text { fewer than } \\
5 \text { years; } \\
\text { microcredit } 5 \\
\text { years or more; } \\
\text { no poor \#\# }\end{array}$ & $\begin{array}{c}\text { Microcredit } \\
\text { non-members }\end{array}$ & $\begin{array}{l}\text { Sexual IPV; deep } \\
\text { interview about } \\
\text { IPV; recall period: } \\
\text { last } 12 \text { months }\end{array}$ & $\begin{array}{l}\text { Women's and } \\
\text { husband's age and } \\
\text { education, occupation } \\
\text { of husband, land } \\
\text { ownership, women's } \\
\text { financial contribution }\end{array}$ \\
\hline Hadi 13 & $\begin{array}{c}\text { Bangladesh } \\
\text { (2005) }\end{array}$ & $\begin{array}{l}\text { Rural villages, } \\
\text { marriage } \\
\text { women }(<50 \\
\text { years); sample } \\
\text { size: } 500\end{array}$ & $\begin{array}{l}\text { Prevalence } \\
\text { of physical } \\
\text { IPV: } 22 \% ; \\
\text { psychological: } \\
28 \% \text { in the past } \\
\text { years }\end{array}$ & $\begin{array}{l}\text { Microcredit } \\
\text { fewer than } \\
5 \text { years; } \\
\text { microcredit } 5 \\
\text { years or more; } \\
\text { no poor \#\# }\end{array}$ & $\begin{array}{c}\text { Microcredit } \\
\text { non-members }\end{array}$ & $\begin{array}{c}\text { Physical and } \\
\text { psychological IPV; } \\
\text { deep interview } \\
\text { about IPV; recall } \\
\text { period: last } 12 \\
\text { months }\end{array}$ & $\begin{array}{c}\text { Women's age and } \\
\text { position, living } \\
\text { standard, age at } \\
\text { marriage, education, } \\
\text { ownership of family }\end{array}$ \\
\hline
\end{tabular}

(continues) 
Table 3 (continued)

\begin{tabular}{|c|c|c|c|c|c|c|c|}
\hline References & $\begin{array}{l}\text { Country } \\
\text { (Year) }\end{array}$ & $\begin{array}{l}\text { Study design/ } \\
\text { Participants/ } \\
\text { Sample size }\end{array}$ & $\begin{array}{c}\text { Baseline } \\
\text { prevalence of } \\
\text { IPV }\end{array}$ & Intervention & $\begin{array}{l}\text { Control } \\
\text { intervention }\end{array}$ & $\begin{array}{l}\text { Type of IPV/ } \\
\text { Measurement } \\
\text { tool/Recall } \\
\text { period }\end{array}$ & $\begin{array}{l}\text { Confounding and } \\
\text { effect modification } \\
\text { variables }\end{array}$ \\
\hline \multicolumn{8}{|l|}{ Microcredit } \\
\hline \multicolumn{8}{|l|}{ Surveys } \\
\hline $\begin{array}{l}\text { Hasan et } \\
\text { al. } 42\end{array}$ & $\begin{array}{c}\text { Bangladesh } \\
\text { (2014) }\end{array}$ & $\begin{array}{l}\text { Rural, urban and } \\
\text { slum villages, } \\
\text { marriage } \\
\text { women with } \\
\text { disabilities (> } 15 \\
\text { years); sample } \\
\text { size: } 226\end{array}$ & $\begin{array}{c}\text { Prevalence } \\
\text { of physical } \\
\text { IPV: } 28,3 \% ; \\
\text { psychological: } \\
41,1 \% \text {; sexual: } \\
18,2 \%\end{array}$ & Microcredit & $\begin{array}{c}\text { Microcredit } \\
\text { non-members }\end{array}$ & $\begin{array}{c}\text { Physical, } \\
\text { psychological } \\
\text { and sexual } \\
\text { IPV combined; } \\
\text { instrument not } \\
\text { mentioned; recall } \\
\text { period: last } 12 \\
\text { months }\end{array}$ & $\begin{array}{c}\text { Women's age, area of } \\
\text { residence, education, } \\
\text { marital status, degree } \\
\text { of disability }\end{array}$ \\
\hline $\begin{array}{l}\text { Karim \& } \\
\text { Law } 43\end{array}$ & $\begin{array}{c}\text { Bangladesh } \\
\text { (2016) }\end{array}$ & $\begin{array}{l}\text { Rural villages, } \\
\text { wife-abusive } \\
\text { marriage men; } \\
\text { sample size: } 243\end{array}$ & $\begin{array}{l}\text { IPV prevalence } \\
\text { not informed }\end{array}$ & $\begin{array}{l}\text { Microcredit } \\
\text { active } \\
\text { participation; } \\
\text { nominal } \\
\text { participation \#\#\# }\end{array}$ & $\begin{array}{c}\text { Microcredit } \\
\text { non-members }\end{array}$ & $\begin{array}{c}\text { Physical, } \\
\text { psychological } \\
\text { and/or sexual IPV } \\
\text { combined; WHO's } \\
\text { questionnaire ** } \\
\text { adaptation; recall } \\
\text { period: last } 12 \\
\text { months }\end{array}$ & $\begin{array}{c}\text { Women's and } \\
\text { husband's education, } \\
\text { marital duration, } \\
\text { household } \\
\text { landholding. Effect } \\
\text { modification: spouse's } \\
\text { conservative ideology }\end{array}$ \\
\hline Kim et al. 37 & $\begin{array}{l}\text { South Africa } \\
\text { (2009) }\end{array}$ & $\begin{array}{l}\text { Rural villages, } \\
\text { marriage } \\
\text { women (> } \\
\text { 50\%) } 18 \text { years } \\
\text { or more; } \\
\text { Protestants: } \\
73,2 \% \text {; sample } \\
\text { size: } 860\end{array}$ & $\begin{array}{l}\text { IPV physical } \\
\text { and or sexual } \\
\text { violence: } 11,4 \% \\
\text { in the past year }\end{array}$ & $\begin{array}{l}\text { IMAGE *: credit } \\
\quad+\text { training + } \\
\text { lecture educator } \\
\text { + ten session } \\
\text { about gender } \\
\text { education }\end{array}$ & $\begin{array}{l}\text { Microcredit } \\
\text { (only cash) }\end{array}$ & $\begin{array}{l}\text { Physical and } \\
\text { sexual IPV } \\
\text { combined; WHO's } \\
\text { questionnaire **; } \\
\text { recall period: last } \\
12 \text { months }\end{array}$ & $\begin{array}{l}\text { Women's age, village } \\
\text { pair, marital status, } \\
\text { lifetime experience } \\
\text { of intimate-partner } \\
\text { violence by current } \\
\text { partner at baseline }\end{array}$ \\
\hline $\begin{array}{l}\text { Koenig et } \\
\text { al. } 33\end{array}$ & $\begin{array}{c}\text { Bangladesh } \\
\text { (2003) }\end{array}$ & $\begin{array}{l}\text { Rural villages, } \\
\text { marriage } \\
\text { women ( } 20 \\
\text { years or more); } \\
\text { sample size: } \\
10,368\end{array}$ & $\begin{array}{c}\text { Physical IPV } \\
\text { prevalence: } \\
42 \%\end{array}$ & $\begin{array}{l}\text { Microcredit } \\
\text { fewer than } \\
2 \text { years; } \\
\text { microcredit } 2 \text { or } \\
\text { more years }\end{array}$ & $\begin{array}{c}\text { Microcredit } \\
\text { non-members }\end{array}$ & $\begin{array}{l}\text { Physical violence; } \\
\text { single question } \\
\text { about IPV; recall } \\
\text { period not } \\
\text { informed }\end{array}$ & $\begin{array}{l}\text { Women's age, } \\
\text { husband's education, } \\
\text { area, number of } \\
\text { living sons, religion, } \\
\text { landholding, family } \\
\text { structure, women's } \\
\text { autonomy index. } \\
\text { Community-level } \\
\text { variable: women's } \\
\text { education and credit } \\
\text { group membership }\end{array}$ \\
\hline $\begin{array}{l}\text { Murshid et } \\
\text { al. } 38\end{array}$ & $\begin{array}{l}\text { Bangladesh } \\
\text { (2015) }\end{array}$ & $\begin{array}{l}\text { Urban and rural } \\
\text { villages; ever- } \\
\text { married women } \\
\text { (15-49 years); } \\
\text { Hindus: 94\%; } \\
\text { sample size: } \\
4,163\end{array}$ & $\begin{array}{c}\text { Physical and/ } \\
\text { or sexual IPV } \\
\text { prevalence: } \\
25 \%\end{array}$ & $\begin{array}{c}\text { BRAC \#, } \\
\text { Grameen } \\
\text { Bank \#, ASA \#, } \\
\text { Proshika \# }\end{array}$ & $\begin{array}{c}\text { Microcredit } \\
\text { non-members }\end{array}$ & $\begin{array}{l}\text { Physical and/ } \\
\text { or sexual IPV } \\
\text { combined; CTS } \\
\text { modified } * * * ; \\
\text { recall period: last } \\
12 \text { months }\end{array}$ & $\begin{array}{l}\text { Women's and } \\
\text { husband's age and } \\
\text { education, age and } \\
\text { education difference, } \\
\text { material assets, } \\
\text { currently employed, } \\
\text { autonomy, decision- } \\
\text { making power. Effect } \\
\text { modification: urban/ } \\
\text { rural population }\end{array}$ \\
\hline
\end{tabular}

(continues) 
Table 3 (continued)

\begin{tabular}{|c|c|c|c|c|c|c|c|}
\hline References & $\begin{array}{c}\text { Country } \\
\text { (Year) }\end{array}$ & $\begin{array}{c}\text { Study design/ } \\
\text { Participants/ } \\
\text { Sample size }\end{array}$ & $\begin{array}{c}\text { Baseline } \\
\text { prevalence of } \\
\text { IPV }\end{array}$ & Intervention & $\begin{array}{c}\text { Control } \\
\text { intervention }\end{array}$ & $\begin{array}{c}\text { Type of IPV/ } \\
\text { Measurement } \\
\text { tool/Recall } \\
\text { period }\end{array}$ & $\begin{array}{l}\text { Confounding and } \\
\text { effect modification } \\
\text { variables }\end{array}$ \\
\hline
\end{tabular}

\section{Microcredit}

Surveys

Murshid 36

Bangladesh (2016)

and rura villages; evermarriage men (15-54 years); sample size:

$$
3,336
$$

$\begin{array}{lcccc}\text { Naved \& } & \text { Bangladesh } & \text { Urban and } & \text { Physical IPV } & \text { Microcredit } \\ \text { Persson 26 } & \text { (2005) } & \text { rural villages; } & \text { prevalence:19\% } \\ & & \text { ever-marriage } & \text { (urban), 15,8\% } \\ & \text { women (15-49 } & \text { (rural) in the } \\ & \text { years); sample } & \text { past year } \\ \text { size: } 2,702 & \end{array}$

\begin{tabular}{|c|c|c|c|c|c|c|c|}
\hline $\begin{array}{l}\text { Sarker et } \\
\text { al. } 30\end{array}$ & $\begin{array}{c}\text { Bangladesh } \\
\text { (2016) }\end{array}$ & $\begin{array}{l}\text { Rural villages, } \\
\text { marriage } \\
\text { women }(<49 \\
\text { years); sample } \\
\text { size: } 180\end{array}$ & $\begin{array}{l}\text { Physical IPV } \\
\text { prevalence: } \\
53,3 \% \text { in the } \\
\text { last } 6 \text { months }\end{array}$ & Microcredit & $\begin{array}{c}\text { Microcredit } \\
\text { non-members }\end{array}$ & $\begin{array}{c}\text { Physical IPV; } \\
\text { instrument not } \\
\text { mentioned; recall } \\
\text { period: last } 6 \\
\text { months }\end{array}$ & $\begin{array}{c}\text { Women's and } \\
\text { husband's age and } \\
\text { education, family } \\
\text { income }\end{array}$ \\
\hline $\begin{array}{l}\text { Schuler et } \\
\text { al. } 12\end{array}$ & $\begin{array}{c}\text { Bangladesh } \\
\text { (1996) }\end{array}$ & $\begin{array}{l}\text { Rural villages, } \\
\text { marriage } \\
\text { women (<50 } \\
\text { years); sample } \\
\text { size: } 710\end{array}$ & $\begin{array}{c}\text { Physical IPV } \\
\text { prevalence: } \\
47 \% \text { in the last } \\
\text { year }\end{array}$ & $\begin{array}{c}\text { Grameen } \\
\text { Bank \#: credit } \\
\text { + non-formal } \\
\text { literacy training; } \\
\text { BRAC \#: credit } \\
\text { + education } \\
\text { and health for } \\
\text { children }\end{array}$ & $\begin{array}{c}\text { Microcredit } \\
\text { non-members }\end{array}$ & $\begin{array}{l}\text { Physical violence; } \\
\text { single question } \\
\text { about IPV; recall } \\
\text { period: last } 12 \\
\text { months }\end{array}$ & $\begin{array}{c}\text { Women's age and } \\
\text { education, number } \\
\text { of surviving sons and } \\
\text { daughters, poverty } \\
\text { status, religion, region, } \\
\text { contribution to family } \\
\text { support }\end{array}$ \\
\hline Vyas et al. 39 & $\begin{array}{c}\text { Tanzania } \\
\text { (2015) }\end{array}$ & $\begin{array}{l}\text { Urban and } \\
\text { rural villages, } \\
\text { marriage or } \\
\text { cohabiting } \\
\text { women }(90 \%) \text {, } \\
\text { mean age of } \\
\text { women: } 30 \\
\text { years; sample } \\
\text { size: } 2,084\end{array}$ & $\begin{array}{c}\text { Physical or/ } \\
\text { and sexual IPV } \\
\text { prevalence: } \\
40 \% \text { (urban) } \\
\text { and 55,6\% } \\
\text { (rural) }\end{array}$ & $\begin{array}{l}\text { Microcredit } \\
\text { cooperative, } \\
\text { microcredit } \\
\text { exclusive } \S\end{array}$ & $\begin{array}{c}\text { Microcredit } \\
\text { non-members }\end{array}$ & $\begin{array}{c}\text { Physical and } \\
\text { sexual violence; } 9 \\
\text { isolated questions } \\
\text { about IPV; recall } \\
\text { period: last } 12 \\
\text { months }\end{array}$ & Women's age \\
\hline
\end{tabular}

(continues)

$\begin{array}{ccc}\begin{array}{c}\text { Physical and/ } \\ \text { or sexual IPV }\end{array} & \text { BRAC\#, } & \text { Microcredit } \\ \text { prevalence: } & \text { Bank\#, ASA \#, } \\ 15 \% & \text { Proshika \# }\end{array}$

Microcredit non-members

a

Physical IPV; CTS questionnaire ***; recall period: last

12 months

\section{Physical and/ or sexual IPV combined; CTS modified *** recall period: last \\ 12 months}

Women's age and education, urban or rural population, intervention media exposure, wealth

assets, current modification: material assets

Women's age and income, husband's education, dowry, in-lows live in the household, respondent relies on natal, family support in crisis, communication between spouses, women's and husband's mother abused by her father, income, muslin, women's attitude toward gender

roles, crime in their community

omen's and husband's age and ucation, family

Women's age and education, number daughters, poverty status, religion, region, support

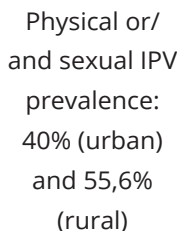
employment. Effect 
Table 3 (continued)

\begin{tabular}{|c|c|c|c|c|c|c|c|}
\hline References & $\begin{array}{l}\text { Country } \\
\text { (Year) }\end{array}$ & $\begin{array}{l}\text { Study design/ } \\
\text { Participants/ } \\
\text { Sample size }\end{array}$ & $\begin{array}{c}\text { Baseline } \\
\text { prevalence of } \\
\text { IPV }\end{array}$ & Intervention & $\begin{array}{c}\text { Control } \\
\text { intervention }\end{array}$ & $\begin{array}{l}\text { Type of IPVI } \\
\text { Measurement } \\
\text { tool/Recall } \\
\text { period }\end{array}$ & $\begin{array}{l}\text { Confounding and } \\
\text { effect modification } \\
\text { variables }\end{array}$ \\
\hline \multicolumn{8}{|l|}{ Cash transfer } \\
\hline \multicolumn{8}{|l|}{ RCT } \\
\hline Angelucci 6 & $\begin{array}{l}\text { Mexico } \\
(2008)\end{array}$ & $\begin{array}{l}\text { Cluster RCT; } \\
\text { rural villages, } \\
\text { women with } \\
\text { child; sample } \\
\text { size: } 12,700\end{array}$ & $\begin{array}{l}\text { IPV prevalence } \\
\text { not available }\end{array}$ & $\begin{array}{l}\text { Oportunidades } \\
\S \S \text { conditional } \\
\text { cash transfer } \\
\text { program: cash } \\
\text { + health and } \\
\text { education for } \\
\text { children }\end{array}$ & $\begin{array}{l}\text { Oportunidades } \\
\text { non-members }\end{array}$ & $\begin{array}{l}\text { Physical IPV; } \\
\text { questionnaire } \\
\text { and recall period } \\
\text { uninformed }\end{array}$ & $\begin{array}{c}\text { Women's and } \\
\text { husband age, } \\
\text { women's education, } \\
\text { household size, natural } \\
\text { disaster, poverty } \\
\text { status, alcohol price. } \\
\text { Interaction: transfer } \\
\text { size and husband with } \\
\text { traditional views of } \\
\text { gender role }\end{array}$ \\
\hline $\begin{array}{l}\text { Hidrobo \& } \\
\text { Fernald } 18\end{array}$ & $\begin{array}{c}\text { Ecuador } \\
\text { (2013) }\end{array}$ & $\begin{array}{c}\text { Cluster RCT; } \\
\text { urban and } \\
\text { rural villages, } \\
\text { marriage } \\
\text { women (45\%); } \\
\text { poor women } \\
\text { with a child; } \\
\text { Catholics: } 74 \% ; \\
\text { sample size: } \\
\text { 1,250 }\end{array}$ & $\begin{array}{l}\text { Physical IPV } \\
\text { prevalence: } \\
\text { 30\% (control } \\
\text { group), 27\% } \\
\text { (Bono de } \\
\text { Desarrollo } \\
\text { Humano } \S \text { ). } \\
\text { Psychological } \\
\text { IPV prevalence: } \\
56 \% \text { (control } \\
\text { group), 52\% } \\
\text { (Bono de } \\
\text { Desarrollo } \\
\text { Humano) }\end{array}$ & $\begin{array}{c}\text { Bono de } \\
\text { Desarrollo } \\
\text { Humano: non- } \\
\text { conditional } \\
\text { cash transfer } \\
\text { program (only } \\
\text { cash) }\end{array}$ & $\begin{array}{c}\text { Bono de } \\
\text { Desarrollo } \\
\text { Humano non- } \\
\text { members }\end{array}$ & $\begin{array}{c}\text { Physical, } \\
\text { psychological } \\
\text { IPV; WHO's } \\
\text { questionnaire } \\
\star \star ; \text { recall period: } \\
\text { unspecific }\end{array}$ & $\begin{array}{l}\text { Age, marital status, } \\
\text { race, husband's } \\
\text { education, indicator } \\
\text { for whether women } \\
\text { has had a child die, } \\
\text { currently pregnant, } \\
\text { asset index, asset } \\
\text { index squared, urban/ } \\
\text { rural, number of } \\
\text { children 0-5 years } \\
\text { old, whether kitchen } \\
\text { is used for sleeping, } \\
\text { province indicators. } \\
\text { Effect modification: } \\
\text { mother education } \\
\text { and mother relative } \\
\text { education }\end{array}$ \\
\hline $\begin{array}{l}\text { Pettifor et } \\
\text { al. } 44\end{array}$ & $\begin{array}{l}\text { South Africa } \\
\text { (2016) }\end{array}$ & $\begin{array}{l}\text { Individual RCT; } \\
\text { rural villages, } \\
\text { girls aged } 13-20 \\
\text { years if they } \\
\text { were enrolled } \\
\text { in school } \\
\text { grades 8-11, } \\
\text { not married } \\
\text { or pregnant; } \\
\text { sample size: } \\
2,448\end{array}$ & $\begin{array}{c}\text { Physical IPV } \\
\text { prevalence: } \\
17 \% \text { in the last } \\
\text { year }\end{array}$ & $\begin{array}{c}\text { Conditional } \\
\text { cash transfer } \\
\text { program: } \\
\text { cash + school } \\
\text { attendance ( } \geq \\
80 \% \text { of school } \\
\text { days per month) }\end{array}$ & $\begin{array}{l}\text { Non-member } \\
\text { microcredit }\end{array}$ & $\begin{array}{c}\text { Physical IPV; } \\
\text { questionnaire not } \\
\text { mentioned; recall } \\
\text { period: last } 12 \\
\text { months }\end{array}$ & $\begin{array}{l}\text { Women's age, } \\
\text { clustering }\end{array}$ \\
\hline
\end{tabular}

(continues) 
Table 3 (continued)

\begin{tabular}{|c|c|c|c|c|c|c|c|}
\hline References & $\begin{array}{l}\text { Country } \\
\text { (Year) }\end{array}$ & $\begin{array}{l}\text { Study design/ } \\
\text { Participants/ } \\
\text { Sample size }\end{array}$ & $\begin{array}{c}\text { Baseline } \\
\text { prevalence of } \\
\text { IPV }\end{array}$ & Intervention & $\begin{array}{c}\text { Control } \\
\text { intervention }\end{array}$ & $\begin{array}{l}\text { Type of IPVI } \\
\text { Measurement } \\
\text { tool/Recall } \\
\text { period }\end{array}$ & $\begin{array}{l}\text { Confounding and } \\
\text { effect modification } \\
\text { variables }\end{array}$ \\
\hline \multicolumn{8}{|l|}{ Cash transfer } \\
\hline \multicolumn{8}{|l|}{ Surveys } \\
\hline Aísa 49 & $\begin{array}{l}\text { Mexico } \\
(2014)\end{array}$ & $\begin{array}{l}\text { Urban and rural } \\
\text { villages, women } \\
\text { with child; } \\
\text { Catholics: } 83 \% ; \\
\text { sample size: } \\
1,917\end{array}$ & $\begin{array}{l}\text { Psychological } \\
\text { IPV prevalence: } \\
70,5 \% \text { to } \\
\text { Oportunidades } \\
\S \S \text { member, 68\% } \\
\text { non-member }\end{array}$ & $\begin{array}{l}\text { Oportunidades } \\
\text { conditional } \\
\text { cash transfer } \\
\text { program: cash } \\
\text { + health and } \\
\text { education for } \\
\text { children }\end{array}$ & $\begin{array}{l}\text { Oportunidades } \\
\text { non-members }\end{array}$ & $\begin{array}{c}\text { An item about } \\
\text { women's power } \\
\text { on contraceptive } \\
\text { choices was } \\
\text { used as a proxy } \\
\text { of psychological } \\
\text { abuse; recall } \\
\text { period: unspecific }\end{array}$ & $\begin{array}{l}\text { Number of member's } \\
\text { family, dependency, } \\
\text { education, } \\
\text { remittances, property, } \\
\text { rooms, telephone, } \\
\text { electric, electronic, } \\
\text { water, car, fuel. Effect } \\
\text { modification: urban/ } \\
\text { rural population }\end{array}$ \\
\hline $\begin{array}{l}\text { Bobonis et } \\
\text { al. } 17\end{array}$ & $\begin{array}{l}\text { Mexico } \\
(2013)\end{array}$ & $\begin{array}{l}\text { Rural villages, } \\
\text { marriage } \\
\text { women (15 } \\
\text { years or older), } \\
\text { physical and/or } \\
\text { sexual; sample } \\
\text { size: } 2,867\end{array}$ & $\begin{array}{l}\text { IPV prevalence: } \\
16 \% ; \\
\text { physical: } 11 \%, \\
\text { sexual: } 9 \%, \\
\text { psychological: } \\
11 \% \text { in the last } \\
\text { year }\end{array}$ & $\begin{array}{l}\text { Oportunidades } \\
\S \S \text { conditional } \\
\text { cash transfer } \\
\text { program: cash } \\
\text { + health and } \\
\text { education for } \\
\text { children }\end{array}$ & $\begin{array}{l}\text { Oportunidades } \\
\text { non-members }\end{array}$ & $\begin{array}{c}\text { Physical, } \\
\text { psychological, } \\
\text { sexual violence } \\
\text { and threat of } \\
\text { physical violence; } \\
15 \text { questions } \\
\text { about IPV based } \\
\text { on CTS **; recall } \\
\text { period: last } 12 \\
\text { months }\end{array}$ & $\begin{array}{l}\text { Women's and } \\
\text { husband's age, race } \\
\text { and education, } \\
\text { household size, } \\
\text { marital status, years of } \\
\text { marriage, IPV history in } \\
\text { women's family. Effect } \\
\text { modification: decision- } \\
\text { making power, expect } \\
\text { gains to marriage, type } \\
\text { of marital relationship }\end{array}$ \\
\hline $\begin{array}{l}\text { Bobonis et } \\
\text { al. } 48\end{array}$ & $\begin{array}{l}\text { Mexico } \\
(2015)\end{array}$ & $\begin{array}{l}\text { Urban and rural } \\
\text { setting, marriage } \\
\text { women (> } 15 \\
\text { years); sample } \\
\text { size: Total N = } \\
\text { 2,867 (survey } \\
\text { 2003), 4,705 } \\
\text { (survey 2006), } \\
\text { 5,800 (survey } \\
2011 \text { ) }\end{array}$ & $\begin{array}{l}\text { Physical and/or } \\
\text { sexual violence } \\
\text { IPV prevalence: } \\
\text { 15,9\% (2003), } \\
\text { 13,7\% (2006); } \\
\text { 10,2\% (2011) }\end{array}$ & $\begin{array}{l}\text { Oportunidades } \\
\S \S \text { conditional } \\
\text { cash transfer } \\
\text { program: cash } \\
\text { + health and } \\
\text { education for } \\
\text { children }\end{array}$ & $\begin{array}{l}\text { Oportunidades } \\
\text { non-members }\end{array}$ & $\begin{array}{l}\text { Physical, sexual, } \\
\text { and psychological } \\
\text { violence; } 16 \\
\text { isolated questions } \\
\text { about IPV; recall } \\
\text { period: last } 12 \\
\text { months }\end{array}$ & $\begin{array}{l}\text { Women and partner's } \\
\text { age, education and } \\
\text { indigenous status, } \\
\text { attainment level, } \\
\text { household size, } \\
\text { cohabiting couple } \\
\text { indicator, years in } \\
\text { union, histories of } \\
\text { spousal abuse in } \\
\text { parental household } \\
\text { during childhood }\end{array}$ \\
\hline Perova 47 & Peru (2010) & $\begin{array}{l}\text { Urban and } \\
\text { rural villages, } \\
\text { marriage } \\
\text { women (15-49 } \\
\text { years); Catholics: } \\
\text { 76\%; sample } \\
\text { size: } 3,904\end{array}$ & $\begin{array}{c}\text { Physical IPV } \\
\text { prevalence: } \\
14 \%, \\
\text { sexual: } 4 \%, \\
\text { psychological: } \\
\text { 15\% in the last } \\
\text { year }\end{array}$ & $\begin{array}{l}\text { Juntos §§: } \\
\text { conditional } \\
\text { cash transfer } \\
\text { program: cash } \\
\text { + health and } \\
\text { education for } \\
\text { children }\end{array}$ & $\begin{array}{l}\text { Juntos non- } \\
\text { members }\end{array}$ & $\begin{array}{l}\text { Physical, } \\
\text { psychological and } \\
\text { sexual violence; } \\
20 \text { isolated } \\
\text { questions about } \\
\text { IPV; recall period: } \\
\text { last } 12 \text { months }\end{array}$ & $\begin{array}{c}\text { Women's age } \\
\text { and education, } \\
\text { health insurance, } \\
\text { cohabitation, paid } \\
\text { employment, } \\
\text { women's family IPV, } \\
\text { electricity, hygienic } \\
\text { restroom, piped } \\
\text { water, family farming, } \\
\text { land, household } \\
\text { material, urban/rural. } \\
\text { Interaction: number } \\
\text { of children, women's } \\
\text { family IPV as a child, } \\
\text { paid job }\end{array}$ \\
\hline
\end{tabular}

(continues) 
Table 3 (continued)

\begin{tabular}{|c|c|c|c|c|c|c|c|}
\hline References & $\begin{array}{c}\text { Country } \\
\text { (Year) }\end{array}$ & $\begin{array}{c}\text { Study design/ } \\
\text { Participants/ } \\
\text { Sample size }\end{array}$ & $\begin{array}{c}\text { Baseline } \\
\text { prevalence of } \\
\text { IPV }\end{array}$ & Intervention & $\begin{array}{c}\text { Control } \\
\text { intervention }\end{array}$ & $\begin{array}{c}\text { Type of IPV/ } \\
\text { Measurement } \\
\text { tool/Recall } \\
\text { period }\end{array}$ & $\begin{array}{l}\text { Confounding and } \\
\text { effect modification } \\
\text { variables }\end{array}$ \\
\hline \multicolumn{8}{|c|}{ Cash transfer } \\
\hline \multicolumn{8}{|l|}{ Surveys } \\
\hline Perova 47 & Peru (2010) & $\begin{array}{c}\text { Urban and } \\
\text { rural villages, } \\
\text { marriage } \\
\text { women (15-49 } \\
\text { years); Catholics: } \\
\text { 76\%; sample } \\
\text { size: } 3,904\end{array}$ & $\begin{array}{l}\text { Physical IPV } \\
\text { prevalence: } \\
14 \%, \\
\text { sexual: } 4 \% \text {, } \\
\text { psychological: } \\
\text { 15\% in the last } \\
\text { year }\end{array}$ & $\begin{array}{l}\text { Juntos §§: } \\
\text { conditional } \\
\text { cash transfer } \\
\text { program: cash } \\
\text { + health and } \\
\text { education for } \\
\text { children }\end{array}$ & $\begin{array}{l}\text { Juntos non- } \\
\text { members }\end{array}$ & $\begin{array}{c}\text { Physical, } \\
\text { psychological and } \\
\text { sexual violence; } \\
20 \text { isolated } \\
\text { questions about } \\
\text { IPV; recall period: } \\
\text { last } 12 \text { months }\end{array}$ & $\begin{array}{c}\text { Women's age } \\
\text { and education, } \\
\text { health insurance, } \\
\text { cohabitation, paid } \\
\text { employment, } \\
\text { women's family IPV, } \\
\text { electricity, hygienic } \\
\text { restroom, piped } \\
\text { water, family farming, } \\
\text { land, household } \\
\text { material, urban/rural. } \\
\text { Interaction: number } \\
\text { of children, women's } \\
\text { family IPV as a child, } \\
\text { paid job }\end{array}$ \\
\hline $\begin{array}{l}\text { Rivera et } \\
\text { al. } 46\end{array}$ & Mexico (2006) & $\begin{array}{c}\text { Urban setting, } \\
\text { marriage } \\
\text { women (> 50\%), } \\
\text { 18-55 year; } \\
\text { sample size: } \\
\text { 2,558 }\end{array}$ & $\begin{array}{c}\text { Physical IPV } \\
\text { prevalence: } \\
18.7 \% \\
\text { (Oportunidades } \\
\S \S) ; 15,6 \% \\
\text { (control } \\
\text { internal), } \\
22 \% \text { (control } \\
\text { external) }\end{array}$ & $\begin{array}{l}\text { Oportunidades } \\
\text { conditional } \\
\text { cash transfer } \\
\text { program: cash } \\
\text { + health and } \\
\text { education for } \\
\text { children }\end{array}$ & $\begin{array}{c}\text { External control: } \\
\text { community } \\
\text { without } \\
\text { Oportunidades } \\
(292) ; \text { internal } \\
\text { control: eligible } \\
\text { in communities } \\
\text { that receive } \\
\text { Oportunidades } \\
\text { but do not } \\
\text { receive the cash }\end{array}$ & $\begin{array}{c}\text { Physical, } \\
\text { psychological, } \\
\text { sexual and } \\
\text { economic } \\
\text { violence; } 20 \\
\text { isolated questions } \\
\text { about IPV; recall } \\
\text { period: last } 12 \\
\text { months }\end{array}$ & $\begin{array}{c}\text { Women's age and } \\
\text { education, control } \\
\text { group, IPV in } \\
\text { childhood, previous } \\
\text { marriage, alcohol } \\
\text { consumption, freedom } \\
\text { of women, decision } \\
\text { expenditure, decision } \\
\text { about have children }\end{array}$ \\
\hline $\begin{array}{l}\text { Tolman \& } \\
\text { Rosen } 45\end{array}$ & USA (2001) & $\begin{array}{l}\text { Urban setting, } \\
\text { single mothers } \\
\text { (18-54 years); } \\
\text { Chistians: 91,4\%; } \\
\text { sample size: } 753\end{array}$ & $\begin{array}{l}\text { Physical IPV } \\
\text { prevalence: } \\
14,9 \% \text { in the } \\
\text { last year }\end{array}$ & $\begin{array}{c}\text { TANF §§: non- } \\
\text { conditional } \\
\text { cash transfer } \\
\text { program (only } \\
\text { cash) }\end{array}$ & $\begin{array}{c}\text { TANF non-me } \\
\text { mbers }\end{array}$ & $\begin{array}{l}\text { Physical violence; } \\
\text { modified CTS ***; } \\
\text { recall period: last } \\
12 \text { months }\end{array}$ & Uninformed \\
\hline
\end{tabular}

ASA: Association for Social Advancement; BRAC: Bangladesh Rehabilitation Assistance Committee; BRDB: Bangladesh Rural Development Board;

CTS: Conflict Tactics Scale; IPV: intimate partner violence; MWEDO: Massai Woman Development Organization; RCT: randomized clinical trials;

TANF: Temporary Assistance for Needy Families; WHO: World Health Organization.

* Name of microcredit intervention;

** Questionnaire suggested by the WHO to measure IPV;

*** Created by Murray A. Straus to measure IPV;

\# Microcredit financial institution;

\#\# Time of women's participation in the program; no poor: women not eligible to microcredit program;

\#\#\# Active participation: women who have microcredit and are involved in microcredit activity; nominal participation: women who have microcredit but are not involved in microcredit activity;

§ Microcredit cooperative: women working together; microcredit alone: woman working alone;

§ Cash transfer programs. 
questionnaires mostly used to measure IPV were the Conflict Tactics Scales (9 studies) and the World Health Organization (WHO) measurement tool (5 studies). Adjustment for women's age and education were most often employed in the multivariate statistical analyses. Women's decision-making power, education, years of participation in the program, and place of residence (urban vs. rural) were tested as effect modifiers often.

\section{Main results}

Table 4 presents a qualitative summary of the studies' main results, according to the type of intervention, study designs and violence.

\section{- Microcredit programs}

Three randomized clinical trial (RCT) 25,28,29 analyzed the impact of MP on IPV. As presented on Table 4, the studies did not find any effect of MP on physical and sexual abuse, and on physical and psychological abuse combined. Nevertheless, the microcredit (IMAGE) seems to be a protective intervention against physical and sexual IPV when studied in tandem 25.

The results from the nine surveys assessing the association between MP and physical violence were heterogeneous, even after a stratified analysis according to IPV baseline prevalence; religion; or place of living. Among these studies, two indicated that including women in MP increased physical

\section{Table 4}

Effect and statistical estimators of the impact of microcredits (MP) and cash transfer programs (CTP) on different types of violence.

\begin{tabular}{|c|c|c|c|c|c|}
\hline Reference & Physical violence & $\begin{array}{l}\text { Psychological } \\
\text { violence }\end{array}$ & Sexual violence & $\begin{array}{l}\text { Physical and/or } \\
\text { sexual violence }\end{array}$ & $\begin{array}{c}\text { Physical and/ } \\
\text { or psychological } \\
\text { violence }\end{array}$ \\
\hline \multicolumn{6}{|l|}{ Microcredit } \\
\hline \multicolumn{6}{|l|}{$\mathrm{RCT}$} \\
\hline Green et al. 28 (2015) & - & - & - & - & $\begin{array}{c}\beta=0.02 ; 95 \% \mathrm{Cl}:-0.1 ; \\
0.14\end{array}$ \\
\hline Pronyk et al. 25 (2006) & - & - & - & $\begin{array}{c}\mathrm{OR}=0.45 ; 95 \% \mathrm{Cl}: 0.23 \\
0.91\end{array}$ & - \\
\hline Tsai et al. 29 (2016) & $\begin{array}{c}\beta=0.091 \text { ( } p \text {-value non- } \\
\text { significant *) }\end{array}$ & - & $\begin{array}{c}\beta=0.379 \text { ( } p \text {-value } \\
\text { non-significant) }\end{array}$ & $\begin{array}{c}\beta=0.118 \text { ( } p \text {-value non- } \\
\text { significant) }\end{array}$ & - \\
\hline \multicolumn{6}{|l|}{ Surveys } \\
\hline Ahmed 41 (2005) & - & - & - & 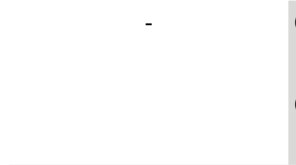 & $\begin{array}{c}\mathrm{OR}=1.47 ; 95 \% \mathrm{Cl}: 0.96 ; \\
2.33 \\
\mathrm{OR}=0.64 ; 95 \% \mathrm{Cl}: 0.25 ; \\
1.66\end{array}$ \\
\hline $\begin{array}{l}\text { Bajracharya \& } \\
\text { Amin } 35 \text { (2013) }\end{array}$ & - & - & - & $\begin{array}{c}\mathrm{RD}=0.018 \text { ( } p \text {-value } \\
\text { non-significant) }\end{array}$ & - \\
\hline Bates et al. 31 (2004) & $\begin{array}{c}\mathrm{OR}=0.75 ; 95 \% \mathrm{Cl}: 0.56 ; \\
1.00\end{array}$ & - & - & - & - \\
\hline Bhuiya et al. 15 (2003) & $\begin{aligned} \mathrm{OR}= & 1.88 ; 95 \% \mathrm{Cl}: \text { not } \\
& \text { informed }\end{aligned}$ & - & - & - & - \\
\hline Cepeda et al. 40 (2017) & - & $\begin{array}{c}\mathrm{OR}=0.70 ; 95 \% \mathrm{Cl}: 0.50 ; \\
0.97\end{array}$ & - & - & - \\
\hline Chin 34 (2012) & - & - & - & $\begin{array}{c}\beta=0.152 \text { ( } p \text {-value non- } \\
\text { significant) }\end{array}$ & - \\
\hline
\end{tabular}

(continues) 
Table 4 (continued)

\begin{tabular}{|c|c|c|c|c|c|}
\hline Reference & Physical violence & $\begin{array}{l}\text { Psychological } \\
\text { violence }\end{array}$ & Sexual violence & $\begin{array}{l}\text { Physical and/or } \\
\text { sexual violence }\end{array}$ & $\begin{array}{c}\text { Physical and/ } \\
\text { or psychological } \\
\text { violence }\end{array}$ \\
\hline
\end{tabular}

\section{Microcredit}

Surveys

Dalal et al. ${ }^{16}$ (2013)

Women with health decision power and primary education: $\mathrm{OR}=1.83 ; 95 \% \mathrm{Cl}: 1.39$;

2.40; secondary education: $\mathrm{OR}=2.74$; 95\%Cl: 2.03; 3.69; high education: $\mathrm{OR}=3.20$; 95\%Cl: 1.62; 6.34

Women without health decision power and primary education: $\mathrm{OR}=1.26 ; 95 \% \mathrm{Cl}$ : $0.88 ; 1.81$; secondary education: $\mathrm{OR}=1.23$; 95\%Cl: 0.81; 1.86; higher education: $\mathrm{OR}=$ 1.47; 95\%Cl: $0.34 ; 6.44$ Dutt et al. 14 (2015) Business cooperative - several women working together: $\beta=-0.13 ; 95 \% \mathrm{Cl}$ : $-0.30 ;-0.02$

Independent owner (-)

Hadi 32 (2000)

Hadi 13 (2005)

Hasan et al. 42 (2014)

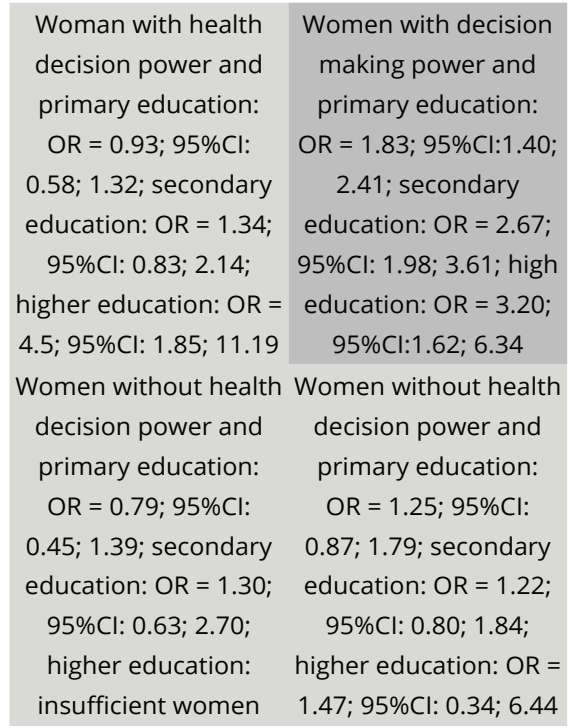

$\begin{array}{cc}\text { Microcredit } & \text { Microcredit } \\ \text { participation less } & \text { participation less } \\ \text { than } 5 \text { years: OR }= & \text { than } 5 \text { years: OR = } \\ 0.75 \text { ( } p \text {-value non- } & 0.56 \text { ( } p \text {-value non- } \\ \text { significant) } & \text { significant) } \\ \text { Microcredit } & \text { Microcredit } \\ \text { participation for } 5 & \text { participation for } 5 \\ \text { years or more: OR }= & \text { years or more: OR }= \\ 0.32 \text { ( } p \text {-value < 0.05) } & 0.35 \text { ( } p \text {-value }<0.05 \text { ) }\end{array}$
Microcredit
participation less
than 5 years: $O R=$
0.79 (p-value non-
significant)
Microcredit
participation for 5
years or more: OR
$=0.65$ ( $p$-value non- significant)

(continues) 
Table 4 (continued)

\begin{tabular}{|c|c|c|c|c|c|}
\hline Reference & Physical violence & $\begin{array}{l}\text { Psychological } \\
\text { violence }\end{array}$ & Sexual violence & $\begin{array}{l}\text { Physical and/or } \\
\text { sexual violence }\end{array}$ & $\begin{array}{c}\text { Physical and/ } \\
\text { or psychological } \\
\text { violence }\end{array}$ \\
\hline
\end{tabular}

\section{Microcredit}

Surveys

Karim \& Law 43 (2016)

Physical, sexual and psychological violence combined.

Active participation: $\beta=-0.194$ ( $p$-value $<0.05$ )

Physical, sexual and psychological violence combined.

Nominal participation: $\beta=-0.095$ ( $p$-value non-significant)

Kim et al. 37 (2009)

\begin{abstract}
$-$
\end{abstract}
Koenig et al. 33 (2003)

Jessore (Bangladesh): $\beta=-1.682$ ( $p$-value non-significant) Sirajgonj (Bangladesh): $\beta=-0.713$ ( $p$-value non-significant)

Murshid et al. 38 (2015)

Murshid 36 (2016)

Naved \& Person 26 (2005)

Sarker et al. 30 (2016)

Schuler et al. 12 (1996)

$$
\begin{gathered}
\text { Urban: } \mathrm{OR}=1.83 \\
\text { ( } p \text {-value }<0.05) \\
\text { Rural: } \mathrm{OR}=1.08 \\
\text { ( } p \text {-value non- } \\
\text { significant) } \\
\text { OR = } 5.4 \text { ( } p \text {-value < } \\
0.05 \text { ) } \\
\text { Grameen Bank **: } \\
\text { OR = 0.30; } 95 \% C l: \\
0.18 ; 0.51 \\
\text { BRAC **: OR = } 0.44 ; \\
\text { 95\%Cl: } 0.28 ; 0.70
\end{gathered}
$$

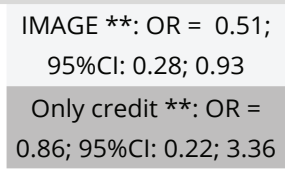

Women with relatively

better economic

status: $\beta=0.47$

( $p$-value $<0.05$ )

Poorer women: $\beta=$

-0.04 ( $p$-value nonsignificant)

$\beta=0.22$ ( $p$-value nonsignificant)

(continues) 
Table 4 (continued)

\begin{tabular}{llccc}
\hline Reference & Physical violence & $\begin{array}{c}\text { Psychological } \\
\text { violence }\end{array}$ & $\begin{array}{c}\text { Sexual violence } \\
\text { sexual violence }\end{array}$ & $\begin{array}{c}\text { Physical and/or } \\
\text { or psychological } \\
\text { violence }\end{array}$
\end{tabular}

\section{Microcredit}

Surveys

BRAC **: OR = 0.44;

95\%Cl: $0.28 ; 0.70$

Vyas et al. 39 (2015)

\section{Cash transfer}

RCT

Angelucci 6 (2008)

Hidrobo \& Fernald 18
(2013)

Hidrobo \& Fernald 18
(2013)

Small values of cash transfer: $\beta=-0.08$ ( $p$-value $<0.05$ )

Higher values of cash transfer and husband with traditional views of gender role: $\beta=$ 0.033 ( $p$-value $<0.05$ )

Women with more than primary schooling: $\beta=$ -0.04 ( $p$-value nonsignificant) No effect (effect measure not informed)

$$
\begin{gathered}
\text { Women with more } \\
\text { than primary } \\
\text { schooling: } \beta=-0.21 \\
\text { ( } p \text {-value }<0.05 \text { ) } \\
\text { Women had less than } \\
\text { primary schooling and } \\
\text { had equal to or higher } \\
\text { education than their } \\
\text { partners: } \beta=0.09 \\
\text { ( } p \text {-value }<0.05)
\end{gathered}
$$

Pettifor et al. 44 (2016) OR $=0.66 ; 95 \% \mathrm{Cl}: 0.59$;

Dar es Salaam

(Tanzania): cooperative - several women working together: $\mathrm{OR}=$ 0.40 ( $p$-value $<0.05$ ) Dar es Salaam (Tanzania): exclusive women working alone: $\mathrm{OR}=1.79 ;(\mathrm{p}$-value $<$ $0.05)$

Mbeya (Tanzania): cooperative - several women working together: $\mathrm{OR}=$ 0.70 (p-value nonsignificant) Mbeya (Tanzania): exclusive - women working alone: $\mathrm{OR}=$ 0.68 ( $p$-value nonsignificant)

(continues) 
Table 4 (continued)

\begin{tabular}{|c|c|c|c|c|c|}
\hline Reference & Physical violence & $\begin{array}{l}\text { Psychological } \\
\text { violence }\end{array}$ & Sexual violence & $\begin{array}{l}\text { Physical and/or } \\
\text { sexual violence }\end{array}$ & $\begin{array}{l}\text { Physical and/ } \\
\text { or psychological } \\
\text { violence }\end{array}$ \\
\hline \multicolumn{6}{|l|}{ Cash transfer } \\
\hline \multicolumn{6}{|l|}{ Surveys } \\
\hline \multirow[t]{2}{*}{ Aísa 49 (2014) } & - & $\begin{array}{c}\text { Urban: } \beta=0.150 \\
\text { (p-value }<0.05)\end{array}$ & - & - & - \\
\hline & & $\begin{array}{l}\text { Rural: } \beta=0.116 \\
\text { (p-value non- } \\
\text { significant) }\end{array}$ & & & \\
\hline $\begin{array}{l}\text { Bobonis et al. } 17 \\
(2013) * * *\end{array}$ & $\begin{array}{c}\beta=-0.070(p-\text { value }< \\
0.05)\end{array}$ & $\begin{array}{c}\beta=0.041 \text { ( } p \text {-value non- } \\
\text { significant) }\end{array}$ & $\begin{array}{c}\beta=-0.066 \text { ( } p \text {-value } \\
\text { non-significant) }\end{array}$ & $\begin{array}{c}\beta=-0.096(p-\text { value }< \\
0.05)\end{array}$ & - \\
\hline $\begin{array}{l}\text { Bobonis et al. } 48 \\
\text { (2015) }\end{array}$ & $\begin{array}{c}\beta=-0.016 \text { ( } p \text {-value } \\
\text { non-significant) }\end{array}$ & $\begin{array}{c}\beta=0.049(p-\text { value }< \\
0.05)\end{array}$ & $\begin{array}{c}\beta=0.037(p-\text { value }< \\
0.05)\end{array}$ & $\begin{array}{c}\beta=-0.032 \text { ( } p \text {-value } \\
\text { non-significant) }\end{array}$ & - \\
\hline \multirow[t]{3}{*}{ Perova 47 (2010) } & $\begin{array}{l}\text { Number of children: } \\
\beta=0.02 \text { ( } p \text {-value non- } \\
\text { significant) }\end{array}$ & $\begin{array}{l}\text { Number of children: } \beta \\
=0.02(p \text {-value }<0.05)\end{array}$ & $\begin{array}{c}\text { Number of children: } \\
\beta=0.00 \text { ( } p \text {-value non- } \\
\text { significant) }\end{array}$ & - & - \\
\hline & $\begin{array}{l}\text { Father use to beat } \\
\text { her mother: } \beta= \\
0.05 \text { (p-value non- } \\
\text { significant) }\end{array}$ & $\begin{array}{l}\text { Father use to beat } \\
\text { her mother: } \beta= \\
0.04 \text { ( } p \text {-value non- } \\
\text { significant) }\end{array}$ & $\begin{array}{l}\text { Father use to beat } \\
\text { her mother: } \beta=0.03 \\
\quad(p \text {-value }<0.05)\end{array}$ & & \\
\hline & $\begin{array}{l}\text { Women have a } \\
\text { cash paid job: } \beta= \\
-0.02 \text { ( } p \text {-value non- } \\
\text { significant) }\end{array}$ & $\begin{array}{l}\text { Woman have a cash } \\
\text { paid job: } \beta=-0.07 \\
\quad(p-\text {-value }<0.05)\end{array}$ & $\begin{array}{l}\text { Woman have a cash } \\
\text { paid job: } \beta=-0.05 \\
\quad(p-\text { value }<0.05)\end{array}$ & & \\
\hline Rivera et al. 46 (2006) & $\begin{array}{l}\text { Internal control: } \mathrm{OR}= \\
1.31 ; 95 \% \mathrm{Cl}: 0.86 ; 2.04 \\
\text { External control: OR = } \\
0.81 ; 95 \% \mathrm{Cl}: 0.62 ; 1.05\end{array}$ & $\begin{array}{l}\text { Internal control: OR = } \\
0.79 ; 95 \% \mathrm{Cl}: 0.56 ; 1.09 \\
\text { External control: OR = } \\
0.75 ; 95 \% \mathrm{Cl}: 0 ; 60 ; 0.93\end{array}$ & $\begin{array}{l}\text { Internal control: OR = } \\
1.58 ; 95 \% \mathrm{Cl}: 0.90 ; 2.77 \\
\text { External control: OR = } \\
0.82 ; 95 \% \mathrm{Cl}: 0.60 ; 1.12\end{array}$ & - & - \\
\hline $\begin{array}{l}\text { Tolman \& Rosen } 45 \\
\text { (2001) }\end{array}$ & $\begin{array}{c}\text { No effect (effect } \\
\text { measure not } \\
\text { informed) }\end{array}$ & - & - & - & - \\
\hline
\end{tabular}

-: not measured; 95\%Cl: 95\% confidence interval; BRAC: Bangladesh Rehabilitation Assistance Committee; OR: odds ratio; RCT: randomized clinical trials; RD: risk difference.

* p-value non-significant: $\mathrm{p}$-value $>0.05$;

** Microcredit intervention program;

$\star \star \star$ There was no effect modification considering decision-making power, expect gains to marriage and type of marital relationship.

Note: light gray (protective factor); gray (no effect); dark gray (risk factor).

IPV 15,30, whereas one indicated decline 12, one suggested no relationship ${ }^{31}$, and five showed effect modification by women's education and decision-making power in marriage 16; period of women's participation in the program 13,32; living in a rural or urban area 26; the municipality of residence 33; and type of microcredit organization (cooperative or individual-based credit) 14 . The seven survey studies assessing the effects of MP on physical and or sexual violence also failed to show a clear picture, not even when stratifying the studies by the characteristics described before. As shown in Table 4, whereas three studies found no effect of MP on these type of IPV 34,35,36, four suggested that the effect was modified by women's education and decision-making power in marriage 16; type of microcredit (with or without gender transformative intervention) 37; women's economic status 38; and different municipalities and business arrangement (cooperative vs. individual-based credit) could influence the results 39 .

Only two surveys assessed the repercussions of MP following psychological violence. One found a protective effect 40 , whereas the other suggested that women attending the program five or more 
years tended to experience less victimization 13. Two studies examined the relationships between MP and sexual violence. Both, failed to show any statistically significant effects of the intervention 16,32. The only survey assessing physical and psychological violence combined as the outcome also did not find any association 41 . Two studies used physical, psychological and sexual violence combined as outcome. One showed that MP decrease violence 42 . While still finding that active participation in an MP could decrease violence in general, the second study showed that a nominal participation - when formally receiving the money but without the autonomy to use it at their will -increased the risk among women with conservative husbands 43 .

\section{- Cash transfer programs}

Three randomized controlled trials evaluated the impact of CTP on different forms of IPV 6,18,44. Angelucci 6 found that receiving a more substantial amount of money increased the risk of physical violence among women with partners holding a more traditional view of gender roles, yet that the intervention was protective when women received smaller amounts, regardless of the partner's cultural background. Pettifor et al. 44 pointed out that being part of the CTP was a protective factor for physical violence among school girls. For Hidrobo \& Fernald 18 , the intervention had a protective effect for psychological violence on women with intermediate or higher education, while the risk increased among those with a lower educational level if they had more years of schooling than their partners.

Five surveys evaluated the impact of CTP on physical violence. Only one suggested that CTP reduces physical violence 17 , while four studies reported no effect at all 45,46,47,48. None of the studies showed an increased risk. The effects of CTP on psychological violence were also covered in five studies. Again, there seems to be no consensus in the literature, even after stratifying the analysis. Two studies showed no impact 17,46; two suggested an effect modification according to place of living (rural vs. urban) 49; number of children, and IPV during childhood and paid job 47; and one showed an increased risk 48 . Four papers studied the effect of CTP on sexual violence 17,46,47,48. Two found no association 17,46, one an increased risk 48 and one a modification effect based on number of children, IPV during childhood and paid job 47.

\section{- Assessment of study quality and sensitive analyses}

Table 1 (experimental studies) and Table 2 (surveys) synthesize the assessment of methodological quality. Focusing solely on RCT studies, criteria 1 (Truly Randomization) and 4 (Intention to Treat Analysis) were endorsed in only one MP 28 and CTP 44 study, respectively. The first paper did not find any association between MP and physical and or psychological violence. The second showed that CTP was protective against physical violence among adolescents.

In surveys, criterion 1 (Random or Pseudo-Random Sample) and 9 (Statistical Analysis) were endorsed in seven MP 26,30,35,36,37,38,43 and three CTP 17,47,48 studies, respectively (Table 2). One of two MP surveys evaluating physical violence 26,30 found a risk effect 30 while the other uncovered a modification effect by place of living 26. Four papers evaluated the effect of MP on physical and or sexual violence 35,36,37,38. Among those, two showed no effect 35,36 and two showed a modification effect by type of intervention 37 and socioeconomic position 38 . The only paper assessing the relation between MP and physical, psychological and sexual violence occurring in tandem revealed a modification effect according to whether women were managing their own businesses 43 .

The CTPs survey papers that endorsed the two methodological criteria had heterogeneous results, too. Those looking at physical violence as the outcome showed a protective effect of CTP 17, but also no effect 47,48. Regarding psychological violence, results were rather mixed. The papers either showed no effect 17, some risk 48 and a modification effect according to the number of children; a previous history of domestic violence in original family; and having or not a paid job 47 . The same heterogeneity persists in sexual violence, as the studies showed no effect 17 , risk 48 and modification effects 47. 


\section{Discussion}

\section{Studies profile}

As shown in the Results section, studies assessing the effect of IPV on women enrolling in economic empowerment programs are restricted to a few countries. Those focusing on MP were restrict to Africa and Southeast Asia, while papers covering CTP were more common in Latin America. The growing interest on the subject is noticeable given more than $50 \%$ of the reviewed papers have been published since 2010. Most studies have focused on MP rather than CTP. The scope of the original studies follows the literature's trend regarding violence against women, which mainly focuses on the assessment of the programs' impact on physical violence.

Methodologically, improvement in the quality of violence identification strategies is perceptible over time, with studies gradually incorporating more robust instruments in recent years. The number of randomized controlled trials remains small compared to surveys. Adequate sample size and the proper use of control groups are positive features found in all original surveys/intervention studies. Variables used to control for confounding were those frequently used in IPV studies. The majority cross-sectional studies disregarded the time of women's participation in economic empowerment programs in the analysis. Nevertheless, the few studies exploring this aspect showed that time from the enrollment in the program is an important facet. Hadi et al. 13, for instance, found that participating in MP for five years or more protects against physical and psychological violence while participating for less than five years bears no effect. Conversely, Bobonis et al. 48 showed that women's participation in income transfer programs could reduce violence initially but that this beneficial effect did not hold over time.

\section{Main results}

The effects of women's enrollment in microcredit and cash transfer programs on physical violence were clearly heterogeneous. Contradictory results came from studies analyzing the impact of MP on physical and or sexual violence as well. The same picture arose when considering the papers focusing on the effect of CTP on psychological and sexual IPV. Conversely, there is some indication that MP is not associated with sexual abuse.

Most studies pointing to an increased risk of physical violence among participants show this association taking place primarily among women with higher education 16, holding more decisionmaking power 16 , living in urban villages 26 and those receiving higher sums of cash 6 . A similar result was reached when physical violence was studied along with sexual abuse. Women with high decisionmaking power 16 and with more years of schooling 16 have an increased risk to IPV. This pattern is also replicated for psychological violence and CTP. Hidrobo \& Fernald 18 showed an increased risk in a small subgroup (women with less than elementary schooling who have more or equal education to their partners). These results suggest that more empowered women are more vulnerable to violence in these specific sets and situations.

Despite some limitations (discussed below), the heterogeneous effects of MP and CTP on IPV uncovered here may be interpreted using the theoretical model proposed by Tauchen et al. 9 . Accordingly, IPV may either increase or reduce following enrollments in economic empowerment programs, depending on the sociocultural context of the family. In countries with a more patriarchal and chauvinist culture, increasing a woman's income could be perceived as a threat to men's power leading to more violence as a result. In contrast, in regions with less gender inequality, economic empowerment may be viewed positively by partners with whom women usually share responsibilities for family support 19 .

These findings underline the importance of considering some peculiarities in attempting to understand the subject, while also showing that there is no single answer to this review question. Moreover, whether physical violence is studied by itself or in tandem with sexual abuse as outcome, the effect of MP may not be generalized to all contexts since most of those studies came from a single country (Bangladesh). 
The literature on the repercussions of MP on levels of psychological violence against women remains scarce, precluding any conclusion. There are only two studies evaluating this relationship 13,40. Compared to research on physical abuse, the small number of studies focusing on psychological violence emphasizes the need to expand the debate.

The few papers studying the association of MP and sexual violence have shown little effect so far 16,32. The difficulty in perceiving a sexual abuse within the marriage may explain this lack of effect, especially in more conservative societies where sexual violence tends to be culturally legitimized. Interventions that explicitly address gender issues and sexuality in marriage would be welcome as strategies aiming to economically empower women and reduce sexual violence.

Besides this general discussion, it is essential to look at the RCT studies in more detail. Focusing on RCTs studying MP, the article published by Pronyk et al. 25, in 2006, is worth mentioning. This is the only study testing a mixed intervention strategy (IMAGE) that coupled MP to professional training, women's follow-up and educational lectures on gender, sexuality, and IPV. As pointed out in the Results section, this initiative proved to be an important protective factor for women regarding partner physical and or sexual violence, either in comparison to women who did not participate in the program or to those receiving only an inductive amount of cash to start a small business 25 . In a separate paper, the authors also pointed out that a single cash intervention was not effective in preventing physical and or sexual violence when the targeted women were compared to those without any intervention 37 , thus strongly indicating the importance of combined strategies - cash, training and health information - when aiming at reducing these types of IPV. Despite these promising results, the unique microcredit RCT that endorsed the quality criteria, showed no effect considering physical and psychological violence combined as the outcome 28.

The RCTs focusing on the impact of CTP on IPV showed a different picture. The effects differed according to the contextual and individual characteristics of women and their partners. Angelucci 6, for instance, identified a protective effect of CTP with respect to physical violence among women who received fewer resources, but pointed out that risk of victimization rose in situations where the amount of cash provided was more substantial. The author showed that this "reverse" pattern occurred, more often, in women with partners holding a more traditional view of gender roles 6 . Pointing out that women benefiting from Oportunidades CTP (Mexico), with low schooling but more education than their partners were at an increased risk of suffering psychological violence as compared to women not included in the program 18. Thus, it shows the importance of considering gender inequalities as a background factor when analyzing the impact of CTP on IPV 18. As also suggested by Tauchen et al. ${ }^{9}$, these findings suggest that men could be using violence as a strategy to "recover" their perceived lost power within the domestic sphere. Conducted by Pettifor et al. 44, the only RCT endorsing both quality criteria, showed a protective effect of CTP on physical violence. Despite this auspicious result, it is important to emphasize that this paper considered physical dating violence and did not analyzed effect modification possibilities.

\section{Other heterogeneity sources}

Even though all programs are based on poverty reduction strategies through money transfer/loan directed to women, the heterogeneity of interventions within and across countries hamper comparisons and thus a comprehensive summarization of the literature. Conditional CTPs, for example, involve actions strengthening women's bonds with society through social support, access to health services and child/adolescent education. The requirement to comply with these demands may lead women to reinforce their social ties and create more favorable conditions for escaping a violent situation or asking for specialized help. This does not occur in non-conditional strategies.

Another source of inconsistency that could not be explored concerns the regional differences in specific objectives, operational strategies and coverage of the CTP programs. For instance, the literature suggests that the programs Oportunidades (conditional CTP/Mexico) and Juntos (unconditional CTP/Ecuador) work very differently 17,18. The contrasting impacts of the initiatives in these countries may be related to their distinct implementation periods, as well as to the different moments the attached conditionalities were monitored 19,20. An impact analysis that does not consider these discrepancies may lead to spurious results. 
The same picture shows up when focusing on MP. In some approaches, complementary actions, such as training and monitoring women's initiatives with a view to their financial autonomy, are part of the program while in several others, women merely receive credit.

Classifying programs based on the presence or absence of actions parallel to the financial incentive is a perspective that could put light in some particularity between microcredit and cash transfer programs. MP has women as the target of all its interventions, in the case of CTPs, women are provided with cash but parallel interventions - the so called conditionalities - have the child and adolescent as the target group. This feature precludes an evaluation of both interventions in tandem. Besides, more than half of the microcredit programs failed to describe the initiative in detail. In many cases it was impossible to figure out all the activities associated with the core intervention (receiving the credit), which precluded any related comprehensive classification.

Another aspect that may have contributed to the inconsistency of the results concerns the differences in outcome measurement strategies. While some studies used cross culturally validated instruments, others opted to remove or add questions from validated questionnaires or, worse, used isolated questions extracted from well-established questionnaires on IPV.

Lastly, regarding the adjustments used in the analyses, most studies included variables classically associated with IPV such as the woman's age, education level or socioeconomic status. However, some studies also adjusted for specific variables seizing local and culturally contoured characteristics, such as the gender of the first child, religion, presence of a dowry, and number of wives. These methodological differences may also hamper a comprehensive synthesis.

\section{Comparing results with previous reviews}

There is a consensus among the reviews conducted by Vyas \& Watts 19, Gibbs et al. 22 and the current study as to the overall impact depending on the specific interventions and individual characteristics, as well as on the social and cultural context wherein the programs are developed. As mentioned before, the effects may diverge according to local, gender norms, women and partner's schooling and other factors. Agreeing with the review advanced by Gibbs et al. 22, the heterogeneity in perspectives, methods and results across studies detected in our study attests to a clear obstacle in implementing a meta-analysis and other strategies aiming at the literature spin-off.

On the other hand, the present study diverges with those from Bourey et al. 20, that indicate that economic interventions are satisfactory approaches to restrain violence against women. This lack of consensus may be caused by the fact that this previous study, as mentioned on the Introduction, had a narrower scope than our review as it was based on only sixteen original researches.

\section{Limitations and strengths}

The results of this review should be interpreted in the light of its limitations and strengths. Restricting the review to papers published in English, Spanish and Portuguese is only a possible limitation. The lack of a formal assessment of publication bias and not carrying the evaluation through to a formal meta-analysis for combining the results were also constraints. Regarding the latter, however, some explanation for not going a step further is due. In fact, we first attempted a quantitative approach, but were impeded not only by the considerable statistical heterogeneity found across studies, but also by the absence of relevant information in the papers for implementing a subgroup analysis. Given most reviewed studies were observational, it was not possible to guarantee the homogeneity of the reference groups regarding possible confounding factors. Regional particularities could not be considered in the analysis as well as other relevant characteristics as accurate description of the intervention, woman and partner's education, number of children per family, amount of microcredit or cash transfer received, intervention period, complementary interventions, family income and religion. Despite these shortcomings, at least three methodological options are a step forward from previous reviews. One concerns the scope of data sources encompassing nine bibliographic databases, as well as the related gray literature. The latter strategy allowed finding 12 papers that were otherwise missed in a previous review with similar scope 20. The three types (and combination) of IPV covered by this review, and the inclusion of both MP and CTP papers are also positive points. 


\section{Literature methodological shortcomings and suggestions to overcome them}

The small number of randomized trials should be mentioned. When dealing with research addressing IPV at core, there is a myth that only observational studies are adequate or a tenable methodological option. However, this is not true 18,25,28. To improve the level of evidence, this type of study design should be encouraged.

The lack of comments on any effect modification in the original papers is another limitation of the literature. As a consequence, we were unable to identify whether this feature was examined yet absent in those studies or if it was assessed at all. Since the literature suggests many variables at the individual and contextual levels modifying the impact of MP/CTP in IPV, reporting these details is welcome to help improving our knowledge about the subject.

The narrow scope of previous literature may also be regarded as a limitation. The role of economic empowerment programs on sexual abuse is one of those issues to be covered. Almost all studies implemented so far have assessed this type of violence along with physical violence precluding more thorough and specific conclusions. Similarly, investing in research to assess effects on psychological violence is required, given former studies are limited to a few countries in Latin America and restricted CTP.

\section{Conclusions}

Microcredit and cash transfer are successful programs for eradicating hunger and poverty on their own right 50,51. However, our results showed that the impact of MP are mixed when it comes to physical and physical/sexual violence, differing markedly according to given scenarios. Even so, the review suggests that the effect of MP on sexual violence is trivial or nonexistent. Regarding the impact of CTPs, the present study showed that the effects on physical, physical/sexual, psychological, and sexual violence were also heterogeneous. Therefore, a single, definitive answer to the main review question-if MP and CTP are associated to IPV prevalence is hence not possible. Although economic empowerment programs for women may increase IPV in some contexts, participation involving not only women but all family members as well should not be discouraged. There is a bulk of evidence that such programs reduce, child mortality, preventable infectious diseases, and other health improvements 52,53,54,55. However, the increase in violence against women should not be ignored. Educational actions encompassing the discussion of gender roles with a focus on eradicating violence against women could be implemented alongside microcredit and income transfer programs in places where there is a high prevalence of IPV.

Beyond the conclusions reached in this article, immanent differences between the types of programs, limitations and heterogeneity of the studies underscore the need of new studies that better account for the cultural contexts and characteristics of the interventions under scrutiny. More specific reviews focusing on the impact of a specific economic empowerment program and a type of IPV should enable a more in-depth account. The increasing interest in the subject in recent years may also help moving forward towards overcoming gaps and limitations faced thus far producing increasingly robust evidence will certainly help better guide the implementation of effective public policies to address this critical global public health problem. 


\section{Contributors}

T. H. Leite contributed to the conception and design, critical review of the intellectual content, and writing of the article. C. L. Moraes, E. S. Marques, R. Caetano, and J. U. Braga contributed to the conception and design and critical review of the intellectual content. M. E. Reichenheim critically reviewed the intellectual content. All authors approved the final version to be published.

\section{Additional informations}

ORCID: Tatiana Henriques Leite (0000-00022861-4480); Claudia Leite de Moraes (0000-00023223-1634); Emanuele Souza Marques (0000-00028633-7290); Rosangela Caetano (0000-0003-14802453); José Ueleres Braga (0000-0001-5247-007X); Michael Eduardo Reichenheim (0000-0001-72326745).

\section{Acknowledgments}

This article was an integral part of the doctoral dissertation of the first author, who obtained funding from the Brazilian Graduate Studies Coordinating Board (Capes) for 39 months.

\section{References}

1. Devries KM, Mak JY, Garcia-Moreno C, Petzold M, Child JC, Falder G, et al. Global health. The global prevalence of intimate partner violence against women. Science 2013; 340: 1527-8.

2. World Health Organization. Understanding and adressing violence against women. Geneva: World Health Organization; 2012.

3. Stromquist NP. Education as a means for empowering women. In: Parpart JL, Rai SM, Staudt KA, editors. Rethinking empowerment: gender and development in a global/local world. London: Routledge; 2002. p. 22-38.

4. World Health Organization. Violence against women: a priority health issue. Geneva: World Health Organization; 1997.

5. Kim J, Watts CH, Hargreaves JR, Ndhlovu LX, Phetla G, Morison LA, et al. Understanding the impact of a microfinance-based intervention on women's empowerment and the reduction of intimate partner violence in South Africa. Am J Public Health 2007; 97:1794-802.

6. Angelucci M. Love on the rocks: domestic violence and alcohol abuse in rural Mexico. Journal of Economic Analysis \& Policy 2008; 8:43.

7. Yunus M, Moingeon B, Lehmann-Ortega L. Building social business models: lessons from the Grameen experience. Long Range Plann 2010; 43:308-25.

8. Forde I, Rasanathan K, Krech R. Public health agencies and cash transfer programmes: making the case for greater involvement. Geneva: World Health Organization; 2011. (Discussion Paper Series on Social Determinants of Health, 4).

9. Tauchen HV, Witte AD, Long SK. Domestic violence: a nonrandom affair. Int Econ Rev (Philadelphia) 1991; 32:491-511.

10. Farmer A, Tiefenthaler J. An economic analysis of domestic violence. Rev Soc Econ 1997; 55:337-58.

11. Bloch F, Rao V. Terror as a bargaining instrument: a case study of dowry violence in rural India. Am Econ Rev 2002; 92:1029-43.

12. Schuler SR, Hashemi SM, Riley AP, Akhter S. Credit programs, patriarchy and men's violence against women in rural Bangladesh. Soc Sci Med 1996; 43:1729-42.

13. Hadi A. Women's productive role and marital violence in Bangladesh. J Fam Violence 2005; 20:181-9.

14. Dutt A, Grabe S, Castro M. Exploring links between women's business ownership and empowerment among Maasai women in Tanzania. Anal Soc Issues Public Policy 2016; 16:363-86.

15. Bhuiya A, Sharmin T, Hanifi SMA. Nature of domestic violence against women in a rural area of Bangladesh: implication for preventive interventions. J Health Popul Nutr 2003; 21:48-54. 
16. Dalal K, Dahlström O, Timpka T. Interactions between microfinance programmes and noneconomic empowerment of women associated with intimate partner violence in Bangladesh: a cross-sectional study. BMJ Open 2013; 3:e002941.

17. Bobonis GJ, González-Brenes M, Castro R Public transfers and domestic violence: the roles of private information and spousal control. Am Econ J Econ Policy 2013; 5:179-205.

18. Hidrobo M, Fernald L. Cash transfers and domestic violence. J Health Econ 2013; 32: 304-19.

19. Vyas S, Watts C. How does economic empowerment affect women's risk of intimate partner violence in low and middle income countries? A systematic review of published evidence. J Int Dev 2009; 21:577-602.

20. Bourey C, Williams W, Bernstein EE, Stephenson R. Systematic review of structural interventions for intimate partner violence in low- and middle-income countries: organizing evidence for prevention. BMC Public Health 2015; 15:1165.

21. Brody C, Hoop TD, Vojtkova M, Warnock R, Dunbar M, Murthy P, et al. Economic selfhelp group programs for improving women's empowerment: a systematic review. Campbell Systematic Reviews 2015; (19).

22. Gibbs A, Jacobson J, Kerr Wilson A. A global comprehensive review of economic interventions to prevent intimate partner violence and HIV risk behaviours. Glob Health Action 2017; 10 Suppl 2:1290427.

23. The Joanna Briggs Institute. The Joanna Briggs Institute Reviewers' Manual 2014. Adelaide: The Joanna Briggs Institute; 2014.

24. Rothman KJ. Epidemiology: an introduction. New York: Oxford; 2012.

25. Pronyk PM, Hargreaves JR, Kim JC, Morison LA, Phetla G, Watts C, et al. Effect of a structural intervention for the prevention of intimate-partner violence and HIV in rural South Africa: a cluster randomised trial. Lancet 2006; 368:1973-83.

26. Naved RT, Persson LA. Factors associated with spousal physical violence against women in Bangladesh. Stud Fam Plann 2005; 36:289300 .

27. Naved RT, Azimb S, Bhuiyaa A, Persson LA. Physical violence by husbands: magnitude, disclosure and help-seeking behavior of women in Bangladesh. Soc Sci Med 2006; 62:2917-29.

28. Green EP, Blattman C, Jamison J, Annan J. Women's entrepreneurship and intimate partner violence: a cluster randomized trial of microenterprise assistance and partner participation in post-conflict Uganda (SSM-D-1401580R1). Soc Sci Med 2015; 133:177-88.

29. Tsai LC, Carlson CE, Aira T, Norcini Pala A, Riedel M, Witte SS. The impact of a microsavings intervention on reducing violence against women engaged in sex work: a randomized controlled study. BMC Int Health Hum Rights 2016; 16:27.
30. Sarker M, Islam S, Ahmed F. Violence against wives in household: a case study in rural Bangladesh. Innovative Issues and Approaches in Social Sciences 2016; 9:43-56.

31. Bates LM, Schuler SR, Islam F, Islam K. Socioeconomic factors and processes associated with domestic violence in rural Bangladesh Int Fam Plan Perspect 2004; 30:190-9.

32. Hadi A. Prevalence and correlates of the risk of marital sexual violence in Bangladesh. J Interpers Violence 2000; 15:787-805.

33. Koenig MA, Ahmed S, Hossain MB, Mozumder ABMKA. Women's status and domestic violence in rural Bangladesh: individual and community level effects. Demography 2003; 40:269-88

34. Chin YM. Credit program participation and decline in violence: does self-selection matter? World Dev 2012; 40:1690-9.

35. Bajracharya A, Amin S. Microcredit and domestic violence in Bangladesh: an exploration of selection bias influences. Demography 2013; 50:1819-43.

36. Murshid NS. Men's response to their wives' participation in microfinance: perpetration and justification of intimate partner violence in Bangladesh. Public Health 2016; 141:14652.

37. Kim J, Ferrari G, Abramsky T, Watts C, Hargreaves J, Morison L, et al. Assessing the incremental effects of combining economic and health interventions: the IMAGE study in South Africa. Bull World Health Organ 2009; 87:824-32.

38. Murshid NS, Akincigil A, Zippay A. Microfinance participation and domestic violence in Bangladesh: results from a nationally representative survey. J Interpers Violence 2015; 31:1579-96

39. Vyas S, Jansen HA, Heise L, Mbwambo J. Exploring the association between women's access to economic resources and intimate partner violence in Dar es Salaam and Mbeya, Tanzania. Soc Sci Med 2015; 146:307-15.

40. Cepeda I, Lacalle-Calderon M, Torralba M. Microfinance and violence against women in rural Guatemala. J Interpers Violence 2017; [Epub ahead of print].

41. Ahmed SM. Intimate partner violence against women: experiences from a woman-focused development programme in Matlab, Bangladesh. J Health Popul Nutr 2005; 23:95-101.

42. Hasan T, Muhaddes T, Camellia S, Selim N, Rashid SF. Prevalence and experiences of intimate partner violence against women with disabilities in Bangladesh: results of an explanatory sequential mixed-method study. J Interpers Violence 2014; 29:3105-26.

43. Karim KMR, Law CK. Microcredit and marital violence: moderating effects of husbands' gender ideology. J Fam Violence 2016; 31:227-38. 
44. Pettifor A, MacPhail C, Hughes JP, Selin A, Wang J, Gomez-Olive FX, et al. The effect of a conditional cash transfer on HIV incidence in young women in rural South Africa (HPTN 068): a phase 3 , randomised controlled trial. Lancet Glob Health 2016; 4:e978-e88.

45. Tolman RM, Rosen D. Domestic violence in the lives of woman receiving welfare: mental health, substance dependence, and economic well-being. Violence Against Women 2001; 7:141-58.

46. Rivera L, Hernández B, Castro R. Asociación entre la violencia de pareja contra las mujeres de las zonas urbanas en pobreza extrema e incorporación al programa de desarrollo humano. In: López MP, Salles V, editors. El Programa Oportunidades examinado desde el género. México DF: Oportunidades/Fondo de Desarrollo de las Naciones Unidas para la Mujer/El Colegio de México; 2006. p. 69-94.

47. Perova E. Buying out of abuse: how changes in women's income affect domestic violence [Doctoral Dissertation]. Berkeley: University of California; 2010

48. Bobonis G, Castro R, Morales JS. Conditional cash transfers for women and spousal violence: evidence of the long-term relationship from the Oportunidades program in Rural Mexico. Washington DC: Inter-American Development Bank; 2015

49. Aísa MG. Conditional cash transfers and intimate partner violence among Mexican couples: the impact of oportunidades on psychological abuse prevalence [Masters Thesis]. Lund: Lund University; 2014.
50. Orton L, Pennington A, Nayak S, Sowden A, White M, Whitehead M. Group-based microfinance for collective empowerment: a systematic review of health impacts. Bull World Health Organ 2016; 94:694-704.

51. Bastagli F, Hagen-Zanker J, Harman L, Barca V, Sturge G, Schmidt T, et al. Cash transfers: what does the evidence say? A rigorous review of programme impact and of the role of design and implementation features. London: Overseas Development Institute; 2016.

52. Carter JD, Daniel R, Torrens AW, Sanchez MN, Maciel ELN, Bartholomay P, et al. The impact of a cash transfer programme on tuberculosis treatment success rate: a quasi-experimental study in Brazil. BMJ Glob Health 2019; 4:e001029.

53. de Souza RA, Nery JS, Rasella D, Guimaraes Pereira RA, Barreto ML, Rodrigues L, et al. Family health and conditional cash transfer in Brazil and its effect on tuberculosis mortality. Int J Tuberc Lung Dis 2018; 22:1300-6.

54. Martins AP, Canella DS, Baraldi LG, Monteiro CA. Cash transfer in Brazil and nutritional outcomes: a systematic review. Rev Saúde Pública 2013; 47:1159-71.

55. Rasella D, Aquino R, Santos CA, Paes-Sousa R, Barreto ML. Effect of a conditional cash transfer programme on childhood mortality: a nationwide analysis of Brazilian municipalities. Lancet 2013; 382:57-64.

56. Moher D, Liberati A, Tetzlaff J, Altman DG; The PRISMA Group. Preferred Reporting Items for Systematic Reviews and MetaAnalyses: The PRISMA Statement. PLoS Med 2009; 6:e1000097. 


\section{Resumo}

A violência entre parceiros intimos (VPI) é um problema de saúde pública de alcance global. Muitas propostas para eliminar a VPI incluem o empoderamento das mulheres através do desenvolvimento socioeconômico individual. Nesse contexto, alguns estudos sugerem que programas de microcrédito $(P M C)$ e de transferência de renda (PTR) também podem reduzir o risco de VPI, enquanto outros apontam para um efeito oposto. Através de uma revisão sistemática, este estudo teve como objetivo investigar a influência do empoderamento econômico das mulheres através de PMCs e PTRs sobre o risco de violência física, psicológica e sexual. A seleção de artigos e documentos foi realizada por dois pesquisadores, com base nos seguintes critérios: publicação em inglês, português ou espanhol; dados primários; avaliação do efeito de PMC ou PTR sobre VPI; casais heterossexuais; mulheres beneficiárias da intervenção; uso de um grupo de comparação elegível para um PMC ou PTR e foco sobre o risco de VPI como o desfecho. Nossos resultados mostraram que o impacto dos PMCs é misto no que diz respeito à violência física e física/sexual. Contanto, a revisão sugere que o efeito dos PMCs sobre a violência sexual é trivial ou inexistente. Quanto ao impacto dos PTRs, o estudo mostrou que os efeitos sobre a violência física, física/sexual, psicológica e sexual também foram heterogêneos. As mulheres mais empoderadas e com alguma autonomia poderiam estar em risco maior. Entretanto, a participação no programa de empoderamento deve ser incentivada para as mulheres e familias pobres. Intervenções paralelas para lider com a VPI devem focar nas principais medidas para reduzir o risco de aumento de prevalência de VPI em determinados cenários.

Violência por Parceiro Íntimo; Programas Governamentais; Empoderamento; Mulheres

\section{Resumen}

La violencia doméstica (VPI por sus siglas en portugués) es un problema de salud pública en todo el mundo. Las propuestas para eliminarla incluyen el empoderamiento de las mujeres a través de su desarrollo socioeconómico. Algunos estudios sugieren que los programas de microcrédito (PMCs) $y$ de transferencia de renta (PTRs) son iniciativas capaces de reducir el riesgo de VPI. Otros estudios indican un efecto contrario. Basándonos en una revisión sistemática, el estudio procuró investigar la influencia del empoderamiento económico de las mujeres, a través de PMCs y PTRs, sobre el riesgo de violencia física, psicológica y sexual. Los artículos y documentos fueron seleccionados por dos investigadores, de acuerdo con los siguientes criterios: estudios publicados en inglés, portugués o español; datos primarios; evaluación del efecto del PMC o PTR sobre la VPI; parejas heterosexuales; mujeres beneficiarias de la intervención; un grupo de comparación elegible para un PMC o PTR $y$ centrados en el riesgo de VPI como desenlace. De acuerdo con nuestros resultados, el impacto de los PMCs es mixto en lo que se refiere a la violencia física y física/sexual. No obstante, la revisión sugiere que el efecto de los PMCs sobre la violencia sexual es trivial o inexistente. En relación con el impacto de los PTRs, el estudio mostró que los efectos sobre la violencia física, física/sexual, psicológica y sexual también son heterogéneos. Las mujeres más empoderadas y con alguna autonomía podrían estar en riesgo. Sin embargo, la participación en el programa de empoderamiento debe incentivarse en el caso de las mujeres y familias pobres. Las intervenciones paralelas para combatir VPI deben dar prioridad a medidas para reducir el riesgo de aumento de la prevalencia de esta violencia en determinados contextos.

Violencia de Pareja; Programas de Gobierno; Empoderamiento; Mujeres
Submitted on $05 / \mathrm{Sep} / 2018$

Final version resubmitted on 24/Apr/2019

Approved on 16/May/2019 